\title{
Parkinson disease-associated cognitive impairment
}

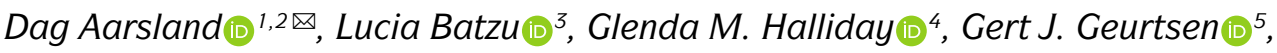 \\ Clive Ballard (106) K. Ray Chaudhuri ${ }^{3}{ }^{3}$ and Daniel Weintraub ${ }^{7,8}$
}

Abstract | Parkinson disease (PD) is the second most common neurodegenerative disorder, affecting $>1 \%$ of the population $\geq 65$ years of age and with a prevalence set to double by 2030 . In addition to the defining motor symptoms of PD, multiple non-motor symptoms occur; among them, cognitive impairment is common and can potentially occur at any disease stage. Cognitive decline is usually slow and insidious, but rapid in some cases. Recently, the focus has been on the early cognitive changes, where executive and visuospatial impairments are typical and can be accompanied by memory impairment, increasing the risk for early progression to dementia. Other risk factors for early progression to dementia include visual hallucinations, older age and biomarker changes such as cortical atrophy, as well as Alzheimer-type changes on functional imaging and in cerebrospinal fluid, and slowing and frequency variation on EEG. However, the mechanisms underlying cognitive decline in PD remain largely unclear. Cortical involvement of Lewy body and Alzheimer-type pathologies are key features, but multiple mechanisms are likely involved. Cholinesterase inhibition is the only high-level evidence-based treatment available, but other pharmacological and non-pharmacological strategies are being tested. Challenges include the identification of disease-modifying therapies as well as finding biomarkers to better predict cognitive decline and identify patients at high risk for early and rapid cognitive impairment.

Parkinson disease (PD) is the most common movement disorder and the second most common neurodegenerative disorder after Alzheimer disease (AD). The neuropathological hallmarks of PD are neuronal loss in the substantia nigra, which causes striatal dopaminergic deficiency, and $\alpha$-synuclein accumulation in intraneuronal inclusions. However, multiple mechanisms and pathway dysfunctions play a role in the pathogenesis of $\mathrm{PD}$, including oxidative stress, dysfunctional mitochondria, cellular calcium imbalance, neuroinflammation and other neurotransmitter system deficits ${ }^{1}$.

Apart from its cardinal motor features, such as bradykinesia (slowness of movement), rigidity and resting tremor, $\mathrm{PD}$ is associated with a heterogeneous spectrum of non-motor symptoms that contribute greatly to the overall disease burden. Cognitive impairment is up to six times more common in individuals with PD than in the healthy population ${ }^{2}$ and is one of the most important non-motor manifestations of PD, integral to the natural history of the disease. Cognitive impairment can severely affect quality of life (QOL) and function and has been shown to have substantial economic consequences over and above the motor symptoms, even at the early stages of $\mathrm{PD}^{3-5}$, thereby representing a high priority for both patients and care partners.
The full spectrum of cognitive impairment occurs in individuals with $\mathrm{PD}$, from subjective cognitive decline (SCD) and mild cognitive impairment (PD-MCI) to dementia (PDD). SCD is a self-perceived decline in cognitive ability, unrelated to an acute event, together with normal age-adjusted, sex-adjusted and education-adjusted performance on standardized cognitive tests ${ }^{6}$. By contrast, PD-MCI is a gradual decline in cognitive ability reported by either a patient with $\mathrm{PD}$ or an informant or observed by the clinician, associated with cognitive deficits on either formal neuropsychological testing or a scale of global cognitive abilities ${ }^{7}$. Subtle difficulties on complex functional tasks may be present ${ }^{7}$ and, based on the number of affected cognitive domains, PD-MCI can be classified as single or multiple domain ${ }^{7}$. PDD is defined as cognitive impairment in a patient with $\mathrm{PD}$ with deficits in at least two of four cognitive domains (executive abilities, attention, visuospatial abilities and memory) that are severe enough to significantly affect normal functioning beyond impairment caused by disease-related motor and autonomic symptoms ${ }^{8,9}$. PDD can be denoted as mild (mild effect on daily functioning), moderate and severe (inability for independent living) dementia. Multiple cognitive domains are affected in those with cognitive impairment and PD, including 


\author{
Author addresses \\ ${ }^{1}$ Department of Old Age Psychiatry, Institute of Psychiatry, Psychology and \\ Neuroscience, King's College London, London, UK. \\ ${ }^{2}$ Centre for Age-Related Medicine, Stavanger University Hospital, Stavanger, Norway. \\ 3Parkinson's Foundation Centre of Excellence, King's College Hospital and Institute \\ of Psychiatry, Psychology and Neuroscience, King's College London, London, UK. \\ ${ }^{4}$ Brain and Mind Centre and Faculty of Medicine and Health School of Medical Sciences, \\ University of Sydney, Sydney, New South Wales, Australia. \\ ${ }^{5}$ Amsterdam UMC, University of Amsterdam, Department of Medical Psychology, \\ Amsterdam Neuroscience, Amsterdam, The Netherlands. \\ ${ }^{6}$ University of Exeter Medical School, Exeter, UK. \\ 'Departments of Psychiatry and Neurology, Perelman School of Medicine at the \\ University of Pennsylvania, Philadelphia, PA, USA. \\ ${ }^{8}$ Parkinson's Disease Research, Education and Clinical Center (PADRECC), Corporal \\ Michael J. Crescenz Veterans Affairs Medical Center, Philadelphia, PA, USA.
}

memory, attention, visuospatial abilities and especially executive functions (mental flexibility, set-shifting, switching, efficiently planning future actions and solving problems $)^{10}$. Of note, dementia with Lewy bodies (DLB) is a disorder characterized by limbic and cortical Lewy body pathology and dementia occurring before or within 1 year after the onset of motor parkinsonism ${ }^{11}$; a specific prodromal, pre-dementia stage has been described $^{12}$. DLB and PDD are thus very similar and distinguished only by the relative timing of motor and cognitive symptoms, although this is under debate ${ }^{13}$.

Despite increased research over the past two decades, the knowledge and treatment of cognitive difficulties in PD lag far behind our knowledge and treatment of its motor features. Continued efforts for a better comprehension of this complex feature of $\mathrm{PD}$ are required, particularly as there is no treatment to prevent or delay cognitive decline in $\mathrm{PD}$, no effective treatments for PD-MCI, and only one treatment (cholinesterase inhibitors) approved for PDD ${ }^{14,15}$.

This Primer describes the epidemiology of $\mathrm{PD}$-associated cognitive impairment and what is known about its mechanisms and pathophysiological changes. In addition, it reviews the diagnostic criteria, procedures and biomarkers to identify patients with PD at increased risk for early and rapid cognitive decline. Finally, this Primer concludes with an overview of the status of pharmacological and non-pharmacological therapeutic strategies and an outline of the most promising breakthroughs that are likely to drive future research pathways.

\section{Epidemiology}

Cognitive decline can occur prior to ${ }^{16}$ or at the time of a diagnosis of PD or a few years or decades after diagnosis and has high variability in its clinical severity, the cognitive domains involved and the rate of progression (FIG. 1). Longitudinal cohort studies have found that people with PD have a 2.5-6-times higher risk of developing dementia than people without PD of similar age $e^{2,17}$. However, the epidemiology of cognitive impairment in $\mathrm{PD}$ is not entirely clear, as population-based studies of PD rarely include PDD or PD-MCI and most studies assess the prevalence and incidence of cognitive impairment in established PD cohorts. In this Primer, we focus on longitudinal studies with relatively large and, when possible, community-based cohorts, using consensus criteria for cognitive impairment classification, based on cognitive testing and clinical interviews.

\section{Dementia}

The cross-sectional proportion of patients with PD who have dementia is $24-31 \%{ }^{18}$. Although findings vary among studies, the cumulative prevalence of PDD in patients with a mean age at diagnosis from 54 to 70.2 years is $17 \%$ at 5 years after diagnosis ${ }^{19}, 46 \%$ at 10 years after diagnosis ${ }^{20}$ and $83 \% 20$ at years after diagnosis $^{21,22}$ (TABLE 1), compared with a global prevalence of $5-7 \%$ of dementia in the general population $>60$ years of age $e^{23}$. Thus, despite variability, there is a high risk of dementia in PD, with nearly half of patients having dementia within 10 years after diagnosis and the vast majority of patients having dementia within $>20$ years after diagnosis. Of note, there is a large variation in time to dementia, as some patients develop dementia within the first few years after diagnosis whereas others remain without dementia for decades. Although several risk factors for cognitive impairment in individuals with $\mathrm{PD}$ have been identified (see 'Risk Factors', below), further understanding of the mechanisms driving this difference and identifying those with a high risk of early dementia to allow for closer monitoring and management is crucial. Importantly, the rate of cognitive decline in PDD is similar to that in $\mathrm{AD}^{24}$ and many patients with PDD will become fully dependent on care and support from others and need nursing home placement ${ }^{25}$.

\section{Mild cognitive impairment}

During the past decade, there has been increased focus on the pre-dementia stages of cognitive impairment in individuals with PD, in particular MCI, as has been the case in $\mathrm{AD}$ and, more recently, also in $\mathrm{DLB}^{12}$. Cross-sectional studies suggest that $25.8 \%$ of patients with $\mathrm{PD}$ without dementia have $\mathrm{MCI}^{26}$, whereas data from the incidence cohort of the ParkWest Study (a prospective longitudinal multicentre study of patients with incident PD in Norway) and other studies found that $\sim 20.2 \%$ of patients have MCI at the time of diagnosis (mean age $71.3 \pm 7.5 \mathrm{SD}$ ), which increases to $40-50 \%$ after 5 years of follow-up ${ }^{27-30}$ (TABLE 1). By contrast, the estimated prevalence of $\mathrm{MCI}$ in the general older population (aged 60-90 years) ranges between $16 \%$ and $20 \%{ }^{31}$.

MCI is often described as a transitory stage between normal cognition (PD-NC) and dementia and it is important to understand the progression from $\mathrm{MCI}$ to dementia. Conversion rates for PDD are markedly increased in those with MCI; for the ParkWest cohort, this was reported to be almost $60 \%$ at 5 years of follow-up for those with PD-MCI ${ }^{27}$. The MCI course is variable and stabilization of cognitive function or even reversion from $\mathrm{PD}-\mathrm{MCI}$ to $\mathrm{PD}-\mathrm{NC}$ has been reported, the latter in approximately $25 \%$ of patients ${ }^{27}$. However, the long-term risk for dementia is still increased in patients with PD who revert from MCI to normal cognition $^{27,32}$. Importantly, the prognostic value of MCI for the development of PDD is influenced by the diagnostic criteria chosen for MCI (see Diagnosis, screening and prevention, below) ${ }^{33}$. 


\section{Subjective cognitive decline}

The emerging concept of $\mathrm{SCD}^{6}$ is also arising from the field of $\mathrm{AD}$ and has been a novel focus of PD research in the past few years. In one of the first studies assessing whether subjective memory complaints in patients with de novo PD (defined as either newly diagnosed patients or patients not receiving dopaminergic medications) could predict cognitive decline, $30.3 \%$ of patients complained of memory issues and were more likely to develop MCI within 2 years of follow-up compared with patients who did not complain of memory issues ${ }^{34}$; subsequent studies have supported this ${ }^{35}$, although several factors, such as affective symptoms ${ }^{36}$, may contribute to progression to MCI (see Diagnosis, screening and prevention, below)

\section{Riskfactors}

Given the wide variation in the time to and rate of cognitive decline in $\mathrm{PD}$, a key research priority is to identify predictors of the future cognitive course for patients with $\mathrm{PD}^{37}$. Several clinical features are associated with an increased risk of cognitive decline and, thus, it is possible to predict the risk for future cognitive impairment or dementia using various algorithms that combine demographic, clinical and genetic features ${ }^{38}$, which may assist the clinician in identifying patients with PD who have a high risk of early dementia. The following predictors, ranked in descending order of weight, were independently associated with the development of cognitive impairment or dementia: presence of hallucinations, older age, overall severity of motor symptoms, presence of speech impairment, older age at PD onset, bradykinesia severity, higher Hoehn and Yahr stage (a descriptive, 5-stage scale commonly used to describe PD severity ${ }^{39}$ ), axial impairment (for example, postural instability and gait difficulty features), a low level of education, presence of depression and male $\operatorname{sex}^{40}$. There are also indications that, in addition to MCI being a risk for dementia, deficits in different cognitive domains may have different predictive power. The CamPaIGN study found that posterior cortical deficits were closely related to incident dementia in $\mathrm{PD}^{41}$. Meanwhile, other studies showed that frontal/executive dysfunction and frontal atrophy were associated with a higher risk for dementia conversion ${ }^{42-44}$. The discrepancies might be ascribed to different genetic or ethnic backgrounds. In the general population, $\sim 40 \%$ of all dementia cases are estimated to be associated with potentially modifiable risk factors, including education, hearing loss, traumatic brain injury, hypertension, diabetes, physical inactivity, excessive alcohol consumption, obesity, smoking, depression, social contact and air pollutants ${ }^{45}$, indicating a potential for prevention. However, it is unclear whether all the risk factors identified for dementia in the general population are also applicable to PDD.

\section{Prodromal PD phenotypes and conversion}

Recent evidence suggests that individuals with prodromal features of PD, such as hyposmia (loss of smell), REM sleep behaviour disorder (RBD) and reduced dopamine transporter binding, may present with worse

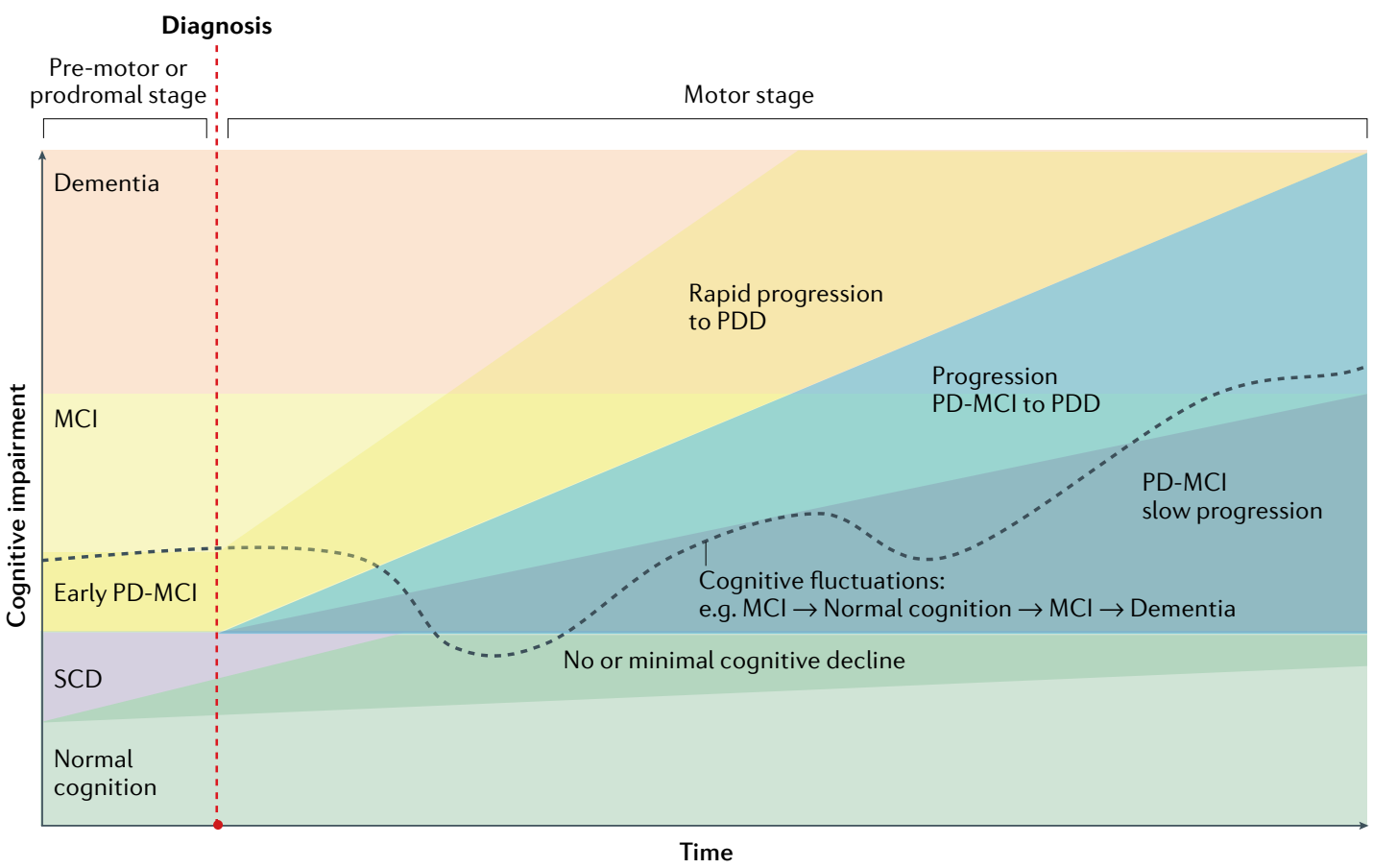

Fig. 1 | The cognitive spectrum and the heterogeneity of progression of cognitive impairment in Parkinson disease. Cognitive changes, mostly in the form of subjective cognitive decline (SCD) or mild cognitive impairment (MCI) can occur prior to or at the time of Parkinson disease (PD) diagnosis or even decades later, with high variability in the rate of progression. Cognitive fluctuations may also occur, in which, for example, some patients with PD-associated $\mathrm{MCl}$ (PD-MCl) may revert to normal cognition and then develop cognitive impairment later in the disease course, typically accompanied by motor progression and the occurrence of other non-motor symptoms. PDD, Parkinson disease dementia. 
cognitive performance compared with people without any or with only one of these features ${ }^{46-48}$. Interestingly, prodromal $\mathrm{PD}$ and $\mathrm{DLB}^{12}$ may overlap and it is not yet known how to distinguish between those who will develop PD versus those who will develop DLB. Of note, cognitive deficit has been recently defined as a new prodromal marker and has been included in the last update of the research criteria for prodromal $\mathrm{PD}^{49}$.

\section{Mechanisms/pathophysiology}

By definition, all patients with PD have the neuropathology of PD with early loss of dopaminergic neurons in the substantia nigra and abnormal deposition of $a$-synuclein in Lewy bodies, initially in cholinergic and monoaminergic brainstem neurons and in the olfactory system, causing significant synaptic pathology $y^{50}$. In patients with coexisting $\mathrm{AD}$ pathology, which is common in and related to cognitive impairment in $\mathrm{PD}, \alpha$-synuclein deposition and synaptic pathology are found in limbic rather than brainstem regions, but the mechanisms of $\alpha$-synuclein proteostasis, degradation and overall prion-like propagation that affects synapses are not thought to differ to those of $\mathrm{PD}^{51}$.

Cognitive decline can occur due to functional brain changes but cognitive decline in PD is thought to relate to neurodegenerative brain changes that differ from those identified in PD-NC. A great variety of theoretical constructs are proposed to underlie the tissue changes associated with cognitive decline in PD, with evidence that multiple degenerative changes and mechanisms may be involved.

\section{Degeneration of neurotransmitter systems}

More widespread dopaminergic deficits in the brain. By definition, all patients with PD have a moderate-tosevere loss of dopaminergic neurons in the nigrostriatal projection pathway. More widespread degeneration of dopaminergic terminals in the striatum - particularly denervation of dopaminergic terminals in the associative dorsal caudate nucleus - occurs in those with PD-MCI than in those with PD without cognitive impairment ${ }^{52}$ (FIG. 2). However, in patients with PD-MCI, there is relative preservation of other dopaminergic systems in the brain ${ }^{52}$, whilst those with PDD have a considerable loss of the lateral dopaminergic system to frontal, parietal and temporal cortical regions ${ }^{52}$ (FIG. 2). In healthy individuals, cortical dopamine modulation can boost working memory as well as visuospatial and attentional processing, and promotes cognitive effort ${ }^{53,54}$, suggesting a key role for dopamine in cognitive function.

\begin{tabular}{|c|c|c|c|c|c|}
\hline Study & $\begin{array}{l}\text { Cohort } \\
\text { selection }\end{array}$ & $\begin{array}{l}n \text { (at } \\
\text { baseline) }\end{array}$ & $\begin{array}{l}\text { Cognitive } \\
\text { outcome }\end{array}$ & Frequency (\%) & Refs \\
\hline $\begin{array}{l}\text { Sydney Multicenter } \\
\text { Study }\end{array}$ & $\begin{array}{l}\text { Research, } \\
\text { de novo }\end{array}$ & 136 & Dementia & $83 \%$ at 20 years & 21 \\
\hline Stavanger Study & Prevalence $^{b}$ & 233 & Dementia & $\begin{array}{l}27 \% \text { at baseline and } 60 \% \text { at } 12 \text { years } \\
(80-90 \% \text { by age } 90)\end{array}$ & 22 \\
\hline \multirow[t]{2}{*}{ Norwegian ParkWest } & \multirow[t]{2}{*}{ Incidence $^{c}$} & \multirow[t]{2}{*}{178} & $\mathrm{MCl}$ & $\begin{array}{l}20.2 \% \text { at baseline, } 28.1 \% \text { at } 1 \text { year, } 38.8 \% \\
\text { at } 3 \text { years and } 43.3 \% \text { at } 5 \text { years }\end{array}$ & 27,277 \\
\hline & & & Dementia & $17.4 \%$ at 4 years & 27 \\
\hline CamPaIGN & Incidence ${ }^{c}$ & 142 & Dementia & $17 \%$ at 5 years and $46 \%$ at 10 years & 19,20 \\
\hline \multirow[t]{2}{*}{ CARPA } & \multirow[t]{2}{*}{$\begin{array}{l}\text { Research, } \\
\text { de novo }^{\mathrm{d}}\end{array}$} & \multirow[t]{2}{*}{123} & $\mathrm{MCl}$ & $\begin{array}{l}35 \% \text { at baseline, } 53 \% \text { at } 3 \text { years and } 50 \% \\
\text { at } 5 \text { years }\end{array}$ & 278 \\
\hline & & & Dementia & $17 \%$ at 5 years & 278 \\
\hline \multirow[t]{2}{*}{ NYPUM } & \multirow[t]{2}{*}{ Incidence $^{c}$} & \multirow[t]{2}{*}{134} & $\mathrm{MCl}$ & $42.6 \%$ at baseline and $72.6 \%$ at 5 years & 29 \\
\hline & & & Dementia & $27.6 \%$ at 5 years & 29 \\
\hline \multirow[t]{2}{*}{ Pennsylvania University } & \multirow[t]{2}{*}{ Convenience ${ }^{\mathrm{e}}$} & \multirow[t]{2}{*}{141} & $\mathrm{MCl}$ & $\begin{array}{l}7.8 \% \text { at } 1 \text { year, } 18.5 \% \text { at } 2 \text { years, } 28 \% \\
\text { at } 3 \text { years, } 36.1 \% \text { at } 4 \text { years and } 43 \% \text { at } \\
6 \text { years }\end{array}$ & 279 \\
\hline & & & Dementia & $\begin{array}{l}0.7 \% \text { at } 1 \text { year, } 3.5 \% \text { at } 2 \text { years, } 7.5 \% \\
\text { at } 3 \text { years, } 12.9 \% \text { at } 4 \text { years and } 28 \% \text { at } \\
6 \text { years }\end{array}$ & 279 \\
\hline ICICLE-PD & Incidence ${ }^{c}$ & 212 & $\mathrm{MCl}$ & $\begin{array}{l}20 \% \text { at baseline }{ }^{f}, 14 \% \text { at } 1.5 \text { years }^{f} \\
\text { and } 16 \% \text { at } 3 \text { years }\end{array}$ & 28 \\
\hline PPMI & $\begin{array}{l}\text { Research, } \\
\text { de novo }^{d}\end{array}$ & 423 & $\begin{array}{l}\mathrm{Cl}(\mathrm{MoCA} \\
<26)\end{array}$ & $\begin{array}{l}21 \% \text { at baseline, } 61.8 \% \text { at } 1 \text { year }^{9}, 69.8 \% \\
\text { at } 2 \text { years } \\
4 \text { years }^{9} \text { and } 68.3 \% \text { at } 3 \text { years } 6 \text {, } 69.9 \% \text { at } 5 \text { years }\end{array}$ & 280 \\
\hline $\begin{array}{l}\mathrm{Cl} \text {, cognitive impairment; } \mathrm{N} \\
\text { cohort. }{ }^{\mathrm{b}} \text { Prevalence comm } \\
\text { cohort. }{ }^{\mathrm{d}} \text { Research cohort, } \mathrm{d} \\
\text { level II diagnostic criteria tc } \\
\text { of patients with symptoms }\end{array}$ & $\mathrm{Cl}$, mild cognitiv & pairment; $\mathrm{N}$ & Montreal Co & $\begin{array}{l}\text { gnitive Assessment. }{ }^{a} \text { De novo university-based } \\
\text { ent community-based population-representa } \\
\text { linic. }{ }^{f} \text { Cumulative prevalence assessed using } \\
\text { elow normative values) for cognitive tests. }{ }^{9 P e r}\end{array}$ & $\begin{array}{l}\text { search } \\
\text { e } \\
\text { dified } \\
\text { ntage } \\
\text { ars. }\end{array}$ \\
\hline
\end{tabular}




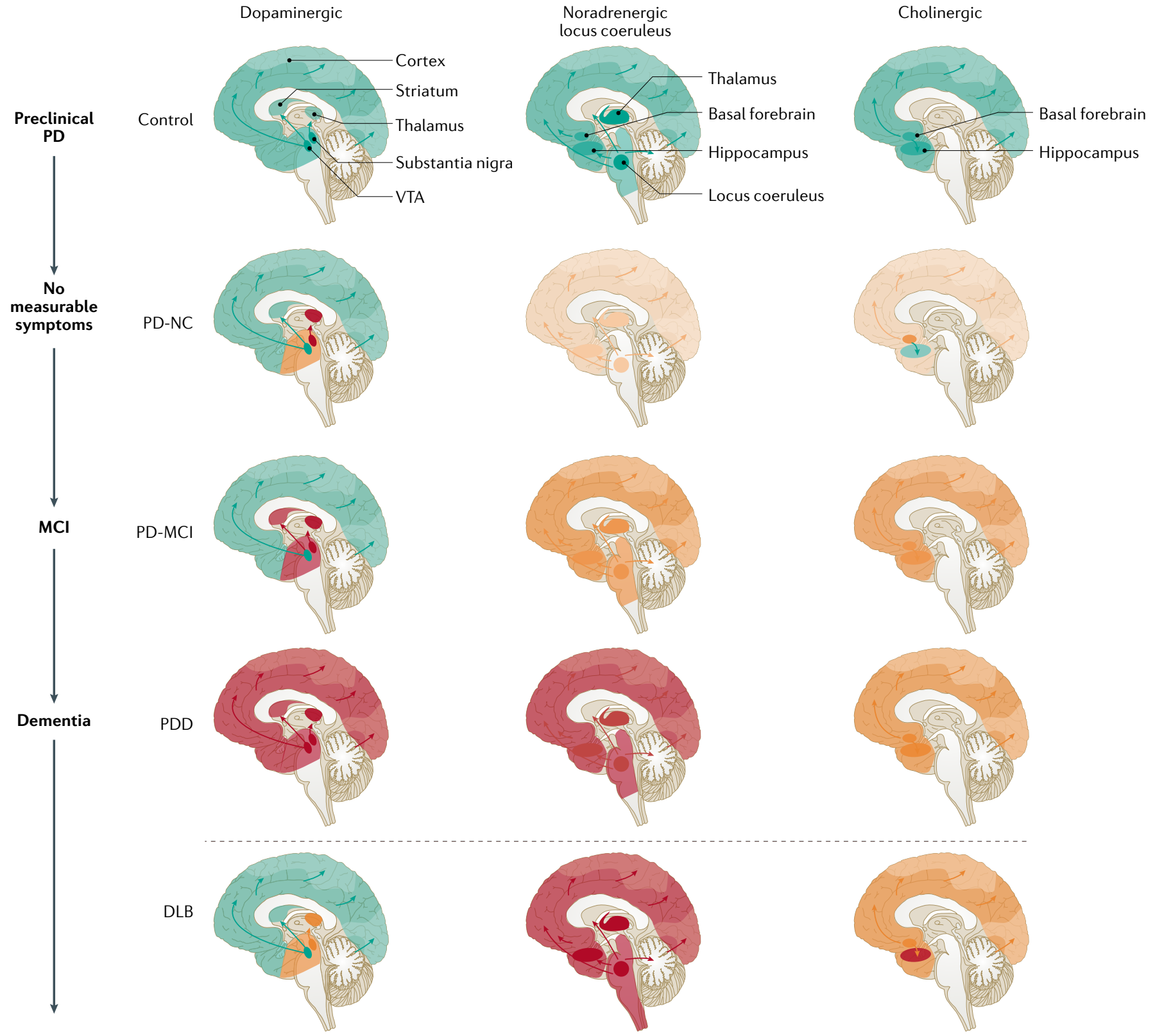

Cell loss and/or denervation

Fig. 2 | Neurotransmitter deficits associated with cognitive decline in PD and DLB. The dopaminergic deficit is widespread initially in the caudate nucleus in Parkinson disease (PD) with mild cognitive impairment (PD-MCl), later progressing to limbic and neocortical brain region in PD dementia (PDD). Dopaminergic deficits are usually more restricted and less severe in dementia with Lewy bodies (DLB). Similar to dopamine, deficits in noradrenaline occur in the brain in PD with normal cognition (PD-NC) but widespread noradrenergic deficits are progressively found with increasing severity of cognitive impairment in PD. Similarly, there are widespread cholinergic deficits in PD-NC but increasing deficits targeting the hippocampus occur with the increasing severity of cognitive decline in PD. Noradrenergic and cholinergic deficits are more severe in DLB. Note that serotonin deficits can occur in PD but are not directly related to cognitive decline. VTA, ventral tegmental area.

Noradrenergic locus coeruleus and sympathetic systems. The locus coeruleus contains noradrenaline-synthesizing neurons that, in humans, produce neuromelanin pigment as a by-product ${ }^{55}$. These neurons promote waking and arousal and are involved in sensory signal detection and modulation of various aspects of cognition but particularly in attention, behavioural flexibility, working and long-term memory ${ }^{56}$. Two areas of dense noradrenergic innervation originating in the locus coeruleus the frontal cortex and hippocampus - are particularly important for cognitive behaviours ${ }^{56}$. At the first diagnosis of $\mathrm{PD}$, there is an association between a reduction in the neuromelanin-sensitive MRI signal of the locus and the presence of $\mathrm{PD}-\mathrm{MCI}^{57}$ (FIC. 2). In addition, there is a 
similar association between a reduction in MRI signal in the locus coeruleus and $\mathrm{RBD}^{58}$ and, in those patients with $\mathrm{PD}$ and $\mathrm{RBD}$, this signal reduction is associated with cognitive deterioration and orthostatic hypotension ${ }^{59}$. Moreover, a reduction in brain noradrenaline transporter availability correlates with cognitive decline and orthostatic hypotension in $\mathrm{PD}^{59}$ and neurogenic orthostatic hypotension in PD owing to noradrenergic denervation of the heart is independently associated with cognitive decline ${ }^{60}$. The underlying mechanism of this association is due to cerebral hypoperfusion caused by orthostatic hypotension, which impairs cognitive function, with noradrenaline-enhancing drugs recommended for the treatment of orthostatic hypotension ${ }^{61,62}$. Of note, the properties of noradrenergic neurons make them more susceptible to oxidative DNA damage compared with other neurons ${ }^{63}$, an increasing problem in patients with reduced blood flow during orthostasis. The evaluation of dopaminergic, noradrenergic and serotonergic markers in CSF and serum in a spectrum of patients with $\mathrm{PD}$ shows increasing alterations in noradrenergic markers that differentiate controls from PD and PD from PDD cases ${ }^{64}$, with only noradrenergic markers significantly reduced in all brain tissue regions from people with $\mathrm{PDD}^{65}$ (FIG. 2). Collectively, data from these studies identify the association of increasing loss of brain noradrenaline and cognitive decline in individuals with PD. On the basis of these data, locus coeruleus imaging and plasma noradrenaline levels are being assessed as potential biomarkers for cognitive decline in a variety of neurodegenerative diseases, including $\mathrm{PD}^{66}$.

Basal forebrain cholinergic systems. The basal forebrain cholinergic neurons are the major source of cholinergic innervation to the neocortex, hippocampus and amygdala ${ }^{67,68}$. These neurons provide important control over circuit dynamics underlying cognitive processing, in particular attention and executive and memory functions ${ }^{67}$. In newly diagnosed patients with $\mathrm{PD}$ and in those further into their disease, a reduction in the volume and density of the basal forebrain cholinergic region and their projections to the neocortex, hippocampus and amygdala is associated with cognitive decline over a 2 -year period ${ }^{69-71}$ and is predictive of cognitive decline in those with PD-NC over 5 years ${ }^{72}$. Of note, the loss of cholinergic fibres is more marked than the loss of cholinergic neurons in $\mathrm{PDD}^{73}$. While the loss of cortical cholinergic innervation is independently associated with cognitive decline in $\mathrm{PD}$, it also interacts with the greater loss of dopamine in the caudate nucleus to contribute to greater cognitive decline $e^{70,74}$. This could be due to the heavy innervation of the basal forebrain cholinergic region by dopamine terminals from midbrain dopaminergic neurons ${ }^{75}$. In terms of memory dysfunction, the loss of basal forebrain cholinergic projections to the hippocampus correlates with memory deficits and conversion to PDD (FIG. 2) ${ }^{71,76}$. Loss of hippocampal cholinergic fibres and activity occurs in patients with PD-MCI, whereas those with PDD have a subsequent increase in $\alpha$-synuclein deposition and dysfunction in both the basal forebrain and hippocampal systems $^{77,78}$.
The mechanisms underlying the degeneration of the basal forebrain cholinergic system are not clear. Unlike the dopaminergic system, the involvement of variations in genes regulating cholinergic function has not been assessed and the cholinergic neurons are not as susceptible to oxidative damage as the noradrenergic locus coeruleus ${ }^{63}$. In addition, increased $\alpha$-synuclein deposition occurs only after the reduction in cholinergic fibres in the cortex ${ }^{78}$ and the widespread aggregation of $\alpha$-synuclein in many neurotransmitter neuronal types does not suggest any selectivity of vulnerability for cholinergic neurons. Of note, there is a selective increase in the innervation of basal forebrain cholinergic neurons by galanin-containing fibres with the development of PD-MCI and progression to PDD, which is thought to be a response to injury, potentially from the cellular increase in $\alpha$-synuclein ${ }^{73}$. This hyperinnervation is lost with increasing cortical AD pathology ${ }^{73}$. Further research is required to determine the mechanisms underpinning the insult to the basal forebrain cholinergic system in PD-MCI.

Serotonergic dysfunction is not directly related to cognitive decline. Although the loss of brainstem serotonergic neurons occurs preclinically and prior to the loss of dopamine neurons in PD, there is no clear relationship between the degeneration of serotonergic neurons and cognitive decline ${ }^{79}$, with both disease progression and older age affecting the severity of degeneration in serotonergic neurons ${ }^{80}$. The degeneration of serotonergic neurons in PD is linked to motor and other nonmotor features such as sleep dysfunction, depression and anxiety $^{81,82}$. In PD, the loss of brain serotonergic structures relates directly to the deposition of $\beta$-amyloid, and medications that increase serotonin neurotransmission reduce $\beta$-amyloid peptide generation and reduce the risk of cognitive decline ${ }^{83}$.

\section{Neuropathology}

In addition to the deposition of $\alpha$-synuclein in Lewy pathologies, other prevalent age-related pathologies can coexist with PD and DLB to affect cognition (FIG. 3) ${ }^{84}$. Of note, neuroinflammation is not a substantive feature of individuals with Lewy pathologies in the absence of $\mathrm{AD}$ pathologies ${ }^{85}$. The most common neuropathology in PDD is limbic and/or neocortical Lewy pathology, with few documented cases without this pathology ${ }^{86}$.

a-synuclein. The abnormal deposition of a-synuclein in vulnerable brainstem and olfactory structures is a definitive feature of PD and can occur prodromally (for example, in those with RBD) and preclinically ${ }^{50,87}$. The question is when and where a-synuclein may have a significant effect on cognition. Atrophy of the entorhinal cortex is associated with memory performance in people with $\mathrm{PD}-\mathrm{MCI}^{88}$ and with $\mathrm{MCI}$ in those without $\mathrm{PD}^{89}$ and, in $\mathrm{PD}$, the density of $\alpha$-synuclein pathology in this region differentiates those progressing to dementia ${ }^{90}$ (FIG. 4). However, the infiltration of $\alpha$-synuclein pathology into limbic (parahippocampal) and neocortical (frontal and temporal association) regions is considered a major determinant of PDD and DLB ${ }^{91}$ (FIG. 4). Indeed, 

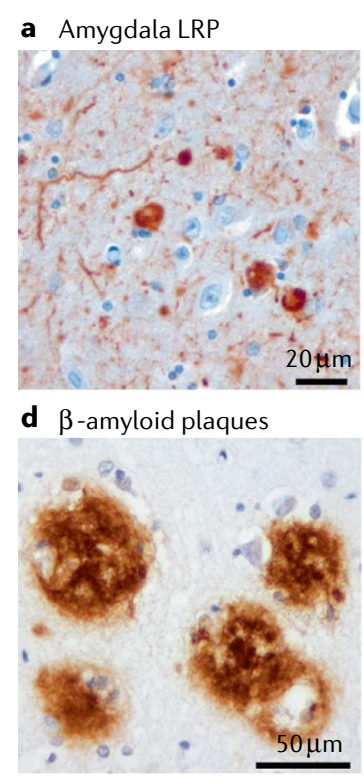

b Cortical Lewy bodies

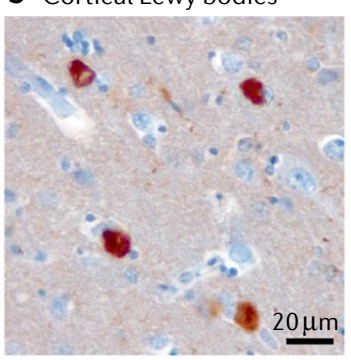

c Striatal LRP

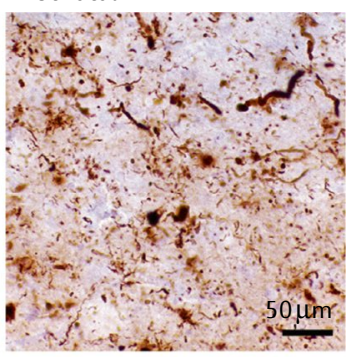

e Tau tangles

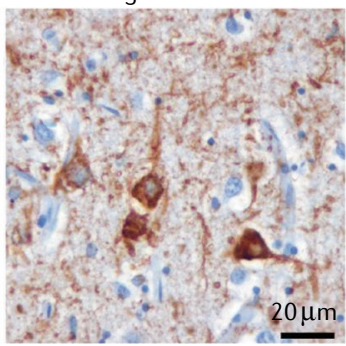

f Amyloid angiopathy

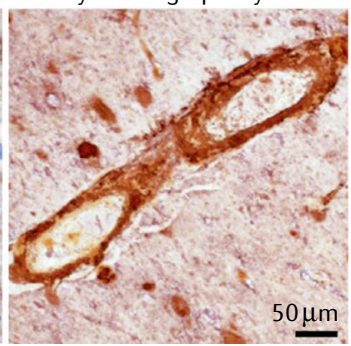

g

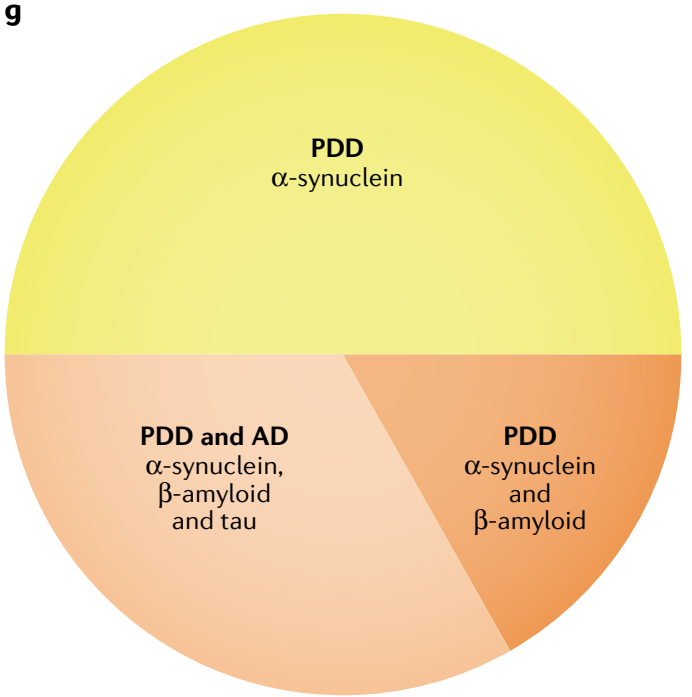

Fig. 3 | The most common neuropathologies associated with PDD. All patients with Parkinson disease dementia (PDD) have $\alpha$-synuclein Lewy pathologies, particularly in medial temporal lobe regions (panel a), but over time there is an increase in neocortical and subcortical Lewy-related pathology (LRP) (panels $\mathbf{b}$ and $\mathbf{c}$ ). Approximately $50 \%$ of patients with PDD have $\beta$-amyloid plaques in the cortex (panel d), which are indicative of pathological changes of Alzheimer disease $(A D)^{290}$. Two-thirds of these patients also have phosphorylated tau deposition (panel e) in cortical tangles indicative of AD, often with amyloid angiopathy (panel $\mathbf{f}$ ) and neuroinflammation ${ }^{86}$ (panel $\mathbf{g}$ ).

individuals with neocortical infiltration of $\alpha$-synuclein pathology have almost twice the yearly decline in cognition compared with those without neocortical infiltration ${ }^{92}$ and a meta-analysis found that neocortical a-synuclein pathology has the strongest association with PDD compared with all other pathologies ${ }^{86}$.

a-Synuclein interacts with neuronal DNA in PD, which may affect DNA repair ${ }^{93}$, and with mitochondria in DLB, drawing mitochondria into $\alpha$-synuclein aggregates and reducing their numbers in cells ${ }^{94}$. This difference in a-synuclein interactions may reflect genetic variation in its coding gene, SNCA, which differs between DLB and $\mathrm{PD}^{95-97}$, thereby affecting the type of SNCA transcripts and $\alpha$-synuclein levels and isoforms in these diseases ${ }^{98}$. These molecular differences in $\alpha$-synuclein between PD and DLB are likely to influence the different types of $\alpha$-synuclein strains that have been documented in these diseases $^{99}$ (FIG. 4). Methods to identify these $\alpha$-synuclein strains in real-time are being standardized ${ }^{100}$; whether these methods will be helpful in predicting cognitive decline in PD remains to be determined.

Other pathology. The most common age-related pathologies in individuals with PD and cognitive impairment are those associated with $\mathrm{AD}$, extracellular $\beta$-amyloid and intracellular tau deposition (FIG. 3). Of note, these pathologies have a different distribution in the brain than Lewy pathologies. One of the earliest age-related pathological markers is the deposition of extracellular $\beta$-amyloid in association cortices; however, it has been shown that the prevalence of positive $\beta$-amyloid PET scans in PD-MCI (5-11\%) is not different to that in aged-matched controls ${ }^{101-103}$, suggesting that the initiation of cognitive decline in PD is not due to significant $\beta$-amyloid deposition. Positive $\beta$-amyloid PET scans precede the substantial tau deposition that together are diagnostic for $\mathrm{AD}^{104}$.

As may be expected by the prevalence of cortical $\beta$-amyloid in PD-MCI, about one-third of patients with PDD have a positive $\beta$-amyloid PET ${ }^{101}$, potentially consistent with the age prevalence of conversion from MCI to $\mathrm{AD}$ over time. In addition, a meta-analysis of more sensitive histological analyses on post-mortem brain tissue found moderate-to-severe extracellular $\beta$-amyloid in cortical and subcortical regions in about half of samples with PDD and severe tau pathology in hippocampal and neocortical regions in around one-third of samples with PDD $^{86}$ (FIG. 3). Coexisting AD pathology in patients with PD increases the amount of limbic and neocortical $\alpha$-synuclein pathology, such that the severity of $\alpha$-synuclein and $\mathrm{AD}$ pathologies are correlated, but also independently predicts progression to $\mathrm{PDD}^{86,105}$. In patients with PDD who have coexisting AD pathology, amyloid angiopathy and neuroinflammation are common and cognitive decline is more rapid with earlier mortality than in PDD without pathological $\mathrm{AD}^{86}$. In addition, patients with $\mathrm{PDD}$ and $\mathrm{AD}$ pathology are older at $\mathrm{PD}$ onset ${ }^{106}$ and have more impaired language than those without coexisting $\mathrm{AD}$, with the severity of language dysfunction (measured with the Boston Naming Test) correlating with increased measures of tau and not of $\beta$-amyloid deposition ${ }^{107}$. Of note, cerebrovascular and TDP43 pathologies do not generally contribute to $\mathrm{PDD}^{86}$.

\section{Genetic factors}

Genetic variation is considered to impact cognition both in $\mathrm{PD}^{108-110}$ and more generally in the population. In terms of the most consistent pathologies, genetic variants 
a

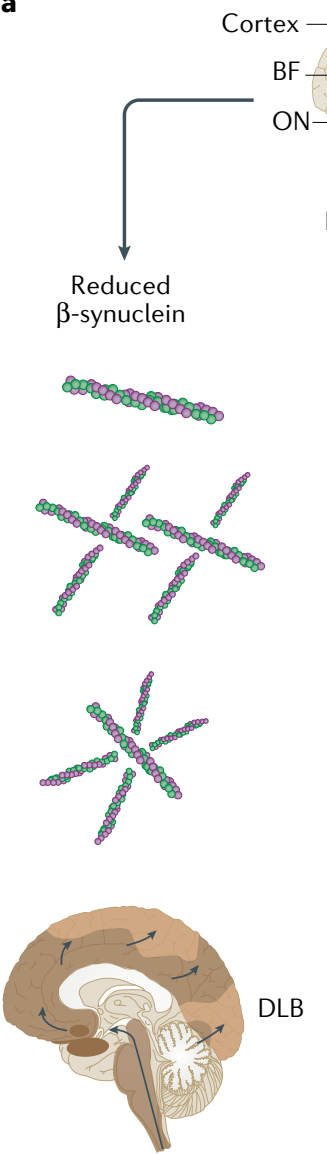

b
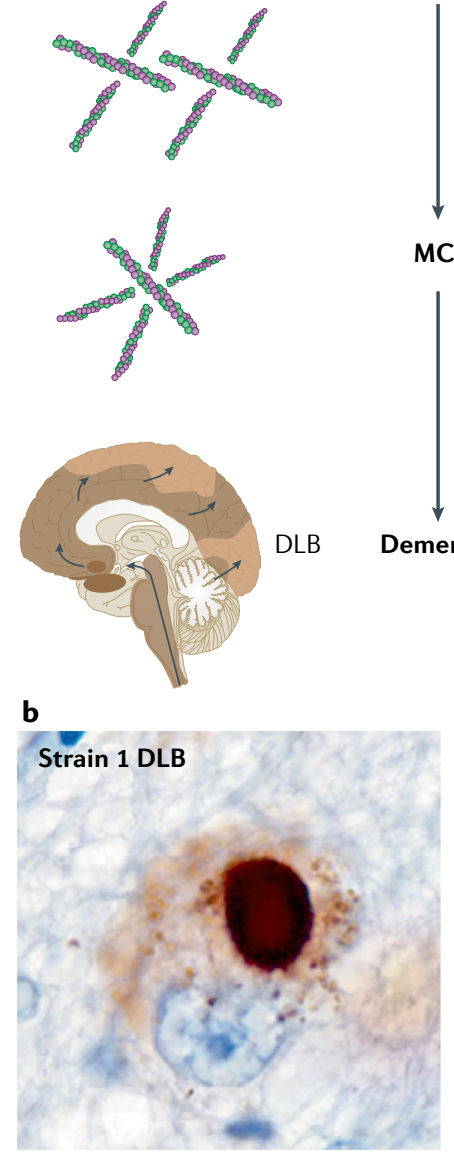

c

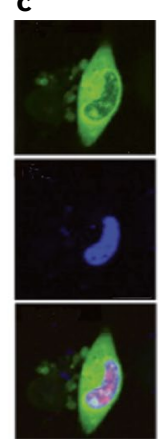

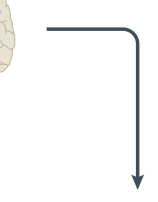

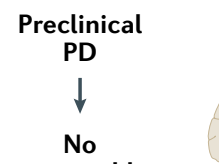
measurable symptoms

$\mathrm{MCl}$
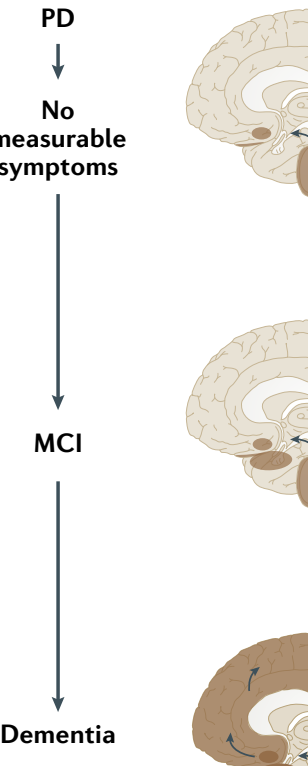

(2)
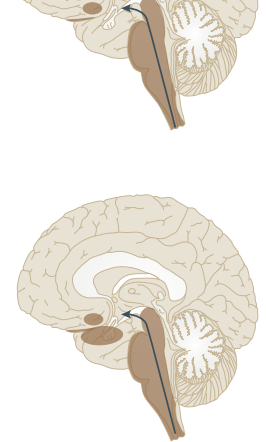

$\mathrm{PD}-\mathrm{MCI}$

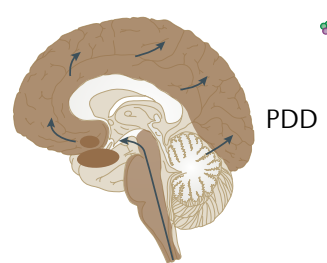

d

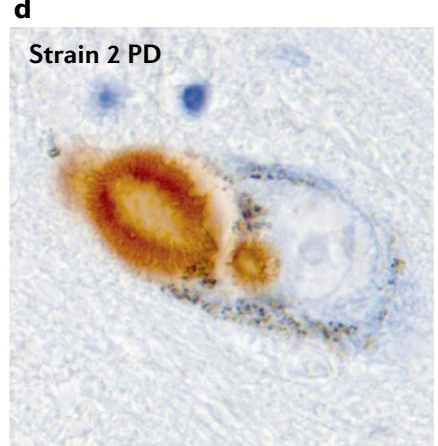

Fig. 4 | Differences in the progression and types of $\alpha$-synuclein pathologies in PD compared with DLB. a | Increasing infiltration of $\alpha$-synuclein pathologies into parahippocampal cortices occurs with increasing cognitive decline in Parkinson disease (PD) but there is also significant infiltration of $\alpha$-synuclein pathology into limbic and neocortical brain regions in both PD dementia (PDD) and dementia with Lewy bodies (DLB).

b | Photomicrograph of $\alpha$-synuclein-labelled Lewy pathologies in cortical neurons in DLB (brown immunoperoxidase, Nissl counterstain). c|a-Synuclein-labelled nucleus and cytoplasm in cell culture (green labelling represents $\alpha$-synuclein, blue labelling represents DAPI (4',6-diamidino-2-phenylindole) staining showing nucleus). d | $\alpha$-Synuclein-labelled Lewy pathologies in cortical neurons in PD (brown immunoperoxidase, Nissl counterstain). In PD, there is evidence that $\alpha$-synuclein interacts with neuronal DNA whereas, in DLB, there is a decrease in $\beta$-synuclein with mitochondria drawn into the $\alpha$-synuclein aggregates (see intracellular dot-like structures in DLB cortical neuron). Genetic variation in $\alpha$-synuclein, $\beta$-synuclein and GBA1 affects the levels, isoforms and pathological seeding capacity of different $\alpha$-synuclein strains documented in PD versus DLB. See REF ${ }^{50}$ for a review of the mechanistic aspects of $\alpha$-synuclein proteostasis, degradation and prion-like propagation. $\mathrm{BF}$, basal forebrain; $\mathrm{H}$, hippocampus; $\mathrm{MCl}$, mild cognitive impairment; ON, olfactory nerve; PD-NC, Parkinson disease with normal cognition; $\mathrm{PD}-\mathrm{MCl}$, Parkinson disease with mild cognitive impairment. Part $\mathbf{c}$ adapted with permission from $\left(\mathrm{REF}^{291}\right)$, Elsevier.

that affect $\alpha$-synuclein levels, the lysosomal potassium channel TMEM175 and the lysosomal metabolism of a-synuclein are also implicated in increased $\alpha$-synuclein pathology in PD and DLB ${ }^{111,112}$. SNCA, TMEM175 and GBA (encoding $\beta$-glucosylceramidase) mutations that respectively increase $\alpha$-synuclein, reduce potassium currents impairing lysosomal and mitochondrial function, and reduce glucocerebrosidase and lysosomal activity are risk factors for both $\mathrm{PD}$ and $\mathrm{DLB}^{112}$. The reduced potassium currents and glucocerebrosidase activity do not result directly in a-synuclein aggregation but respectively increase the phosphorylation of $\alpha$-synuclein and cellular susceptibility to pathological $\alpha$-synuclein seeds (FIG. 4) ${ }^{112,113}$. A particular single nucleotide polymorphism in $G B A$ that reduces glucocerebrosidase expression, weakens its enzymatic activity and enhances $\alpha$-synuclein deposition is associated with PD-MCI and PDD ${ }^{114}$.

Progression and increased cognitive impairment in PD are associated with the APOE (encoding apolipoprotein E) $\varepsilon 4$ allele and with no other genetic variants at the genome-wide level ${ }^{108-110}$. The APOE $\varepsilon 4$ allele may predispose to $\beta$-amyloid deposition over time in these individuals as occurs in the general population.

Poorer cognition and reduced dopamine transmission in the general population has also been associated with genetic variation in SLC6A3 (also known as DAT, encoding dopamine transporter) ${ }^{115}$ as well as in genes involved in dopamine synthesis $(D D C$, encoding dopamine decarboxylase $)^{116}$, degradation enzymes (COMT, encoding catecholamine-O-methyltransferase $)^{117,118}$ and dopamine receptors ( $D R D 2$, encoding dopamine receptor 2$)^{119}$. Collectively, these studies suggest that common genetic variations in a variety of proteins important for the production, metabolism and signalling of dopamine in the brain may predispose to cognitive deficits in PD.

\section{Diagnosis, screening and prevention Diagnosis}

The accurate diagnosis of cognitive impairment in individuals with $\mathrm{PD}$ is important for clinical management, patient information and counselling, and research, including trial selection. The diagnosis can be made based on the evaluation of global cognitive function or on a more detailed neuropsychological assessment, which allows the assessment of attention, working memory, and executive, language, memory and visuospatial functions. A full medical examination is mandatory and biomarkers can be useful to identify the causes and predict the risk of cognitive decline, although their use in clinical practice is yet to be validated.

Screening. Screening of cognitive function in patients with PD is not performed regularly but should be part of routine clinical care. This screening requires less time, fewer resources, is more available and is less burdensome for patients compared with detailed neuropsychological assessments. Disadvantages of screening include limited information about the cognitive profile and reduced reliability compared with neuropsychological assessment.

Based on their clinimetric properties in $\mathrm{PD}^{120}$, three scales for screening of cognitive function were recommended in a paper by the Movement Disorder 
Society (MDS) Rating Scales Review Committee (the Montreal Cognitive Assessment (MoCA) ${ }^{121}$, Mattis Dementia Rating Scale Second Edition (MDRS-2) ${ }^{122}$ and Parkinson's Disease - Cognitive Rating Scale $\left.(\mathrm{PD}-\mathrm{CRS})^{123}\right)$ and two scales were classified as recommended with caveats (Mini-Mental Parkinson $(\mathrm{MMP})^{124}$ and Scales for Outcomes in Parkinson's Disease-Cognition (SCOPA-COG) $\left.)^{125}\right)^{120}$. Although the Mini-Mental State Examination (MMSE) is frequently used, it is not suitable for cognitive screening in the early stage of PD owing to a ceiling effect ${ }^{126}$ and the lack of sensitivity in detecting MCI.

The MoCA is the most frequently used screening instrument in $\mathrm{PD}$ research and clinical practice. The optimal cut-off point of $23 / 24$ has a sensitivity of 0.41 and a specificity of 0.82 , with $68 \%$ correct diagnoses of $\mathrm{PD}-\mathrm{MCI}^{127}$. Based on the individual MoCA score or course of successive MoCA scores, a detailed neuropsychological assessment can be indicated, balancing this cut-off score with other factors such as education, previous level of functioning and availability of neuropsychological assessment.

Neuropsychological testing. Neuropsychological tests are validated standardized tests with adequate population norms. Raw test scores are especially influenced by age and education level. Based on the norms, raw test scores are transformed, correcting for influences, such as age and education, into z-scores or equivalents. Tests are most frequently divided into five domains (attention and working memory, executive, language, memory, and visuospatial functions). Examples of tests that are useful are provided in the MDS consensus PD-MCI paper7. There is large heterogeneity in the neuropsychological tests used in clinical practice. A study using pooled data

\section{Box $1 \mid$ Movement Disorder Society PD-MCl diagnostic criteria ${ }^{7,120}$}

\section{Level I-Abbreviated assessment}

- Impairment on Parkinson disease (PD)-appropriate global cognitive ability scale (such as Montreal Cognitive Assessment (MoCA), Parkinson's Disease - Cognitive Rating Scale (PD-CRS), Mattis Dementia Rating Scale Second Edition (MDRS-2))

- Impairment on at least two neuropsychological tests when a limited set of tests is used (less than two tests per domain or less than five cognitive domains assessed)

\section{Level II - Comprehensive assessment}

- Neuropsychological testing includes two tests per domain:

- Attention and working memory

- Executive functions

- Language

- Memory

- Visuospatial skills

- Impairment on two tests in one domain or impairment on one test in two different domains

- Impairment shown by:

Score 1-2 SD below norm

Significant decline on serial testing

Significant decline from estimated premorbid functioning

PD with mild cognitive impairment (PD-MCI) subtype classification (comprehensive level II assessment required)

- Single domain: impairment on two or more tests in one domain

- Multiple domain: impairment on at least one test in each of two or more domains across multiple international sites could not recommend with confidence a test battery that would be sensitive to detect mild cognitive deficits in patients with $\mathrm{PD}^{128}$. Therefore, the selection of tests should be done based on the presence of adequate local population norms.

Subjective and mild cognitive impairment. Research in healthy older adults has suggested that subjectively identified cognitive decline may indicate early changes in cognitive functioning that are not detected on neuropsychological tests. SCD can be reported by the patient, family members or friends, or clinicians. To our knowledge, there are no validated instruments to determine the presence of SCD in PD. However, tools developed for the assessment of non-motor symptoms, such as the Non-Motor Symptoms Scale (NMSS) ${ }^{129}$, the MDS Non-Motor Rating Scale (MDS-NMS) ${ }^{130}$ and the Non-Motor Symptoms Questionnaire (NMSQ) ${ }^{131}$, include questions on the cognitive status as perceived by the patient. Nevertheless, the value of subjective cognitive complaints in patients with PD without objective impairment in formal neuropsychological testing is not well understood. Although the presence of SCD does not correspond well with objective cognitive impairment, it represents an increased risk for cognitive decline in some but not all studies ${ }^{34,35}$. Of note, as SCD can be due to anxiety or depression, screening and treatment of depression and anxiety is important in patients with subjective cognitive impairment in addition to the monitoring of cognitive function.

Diagnostic criteria for PD-MCI from the MDS classify PD-MCI as SCD reported by patient, caregiver or clinician and impairments at neuropsychological assessment that do not significantly interfere with functional independence (BOX 1). A detailed patient interview is essential to differentiate the effects of cognitive and motor impairment on functioning and to distinguish between MCI and dementia. This can be done, for example, with the Parkinson's Disease Cognitive Functional Rating Scale (PD-CFRS) ${ }^{132}$ or Penn Parkinson's Daily Activities Questionnaire-15 (PDAQ-15) ${ }^{133}$. The PD-MCI criteria contain a two-level operational scheme of PD-MCI depending on the comprehensiveness of the clinical assessment, in which level I is based on an abbreviated assessment (such as screening of cognitive function or a limited battery of neuropsychological tests) and level II is based on comprehensive neuropsychological testing of five cognitive domains (BOX 1). The MDS PD-MCI criteria appeared to have prognostic validity for the development of $\mathrm{PD}$ dementia with both the level I limited test battery ${ }^{134}$ and level II criteria ${ }^{135}$. In a meta-analysis, level I criteria were associated with a greater reversion estimate from PD-MCI to normal cognitive functioning ${ }^{136}$. However, different cut-offs for PD-MCI in neuropsychological testing, different global scales for cognitive screening and the limited battery of neuropsychological tests were all treated equally as level I in this meta-analysis. Therefore, we do not know precisely which level I operationalization led to the greater reversion. Overall, the sensitivity and specificity of level I testing are probably less adequate than those of level II testing, leading to 


\section{Box 2 | Diagnostic procedure Movement Disorder Society PDD criteria ${ }^{8,144}$}

Level I - Parkinson disease dementia (PDD)

- A diagnosis of Parkinson disease (PD) based on the UK Brain Bank criteria for PD

- PD developed prior to the onset of dementia

- Mini-Mental State Examination (MMSE) below 26

- Cognitive deficits severe enough to impact daily living (caregiver interview or Pill Questionnaire) independent of motor symptoms

- Impairment in more than one cognitive domain, that is, at least two of the following aspects:

- Months Reversed or Seven Backward

- Lexical Fluency or Clock Drawing

- MMSE Pentagons

Three-Word Recall

- Absence of major depression

- Absence of delirium

- Absence of other abnormalities that obscure diagnosis

Level II - Comprehensive assessment for characterizing PDD

The level Il evaluation assesses four domains:

- Decreased global cognitive efficiency

- Subcorticofrontal features of PDD

- Instrumental (cortically mediated) functions:

- Language

- Visuoconstructive

- Visuospatial

- Visuoperceptive

- Neuropsychiatric features:

- Apathy

- Depression

- Visual hallucination

- Psychosis
Functional impairment due to cognitive impairment is essential and, as mentioned above, can be identified using the PD-CFRS and PDAQ-15 or during a clinical interview with the patient and an informant. Also similar to the PD-MCI criteria, the PDD criteria contain a two-level operational scheme depending on the comprehensiveness of the clinical assessment (BOX 2).

In dementia trials in $\mathrm{PD}$, other rating scales have been used to assess the degree of cognitive impairment, its effect on activities of daily living and the clinical global impression of change, although none of them have been specifically designed nor recommended for PDD. These include the Alzheimer's Disease Assessment Scale Cognitive Subscale (ADAS-Cog) ${ }^{138}$, the Alzheimer's Disease Cooperative Study - Activities of Daily Living Scale (ADCS-ADL) ${ }^{139}$ and the Alzheimer's Disease Cooperative Study - Clinical Global Impression of Change (ADCS-CGIC) ${ }^{140}$, all of which have been developed in the context of dementia due to AD.

In the context of a diagnosis of PDD, it is important to rule out other causes of cognitive impairment such as concomitant physical disease, drug use, depression or delirium. In addition to physical examination and history, basic blood tests (for example, thyroid function tests, vitamin B12 level and relevant tests for metabolic, infectious, autoimmune and other aetiologies) ${ }^{141}$ and structural brain imaging with MRI should be performed to rule out other causes such as severe cerebrovascular disease. PDD is also associated with atrophy in brain $\mathrm{MRI}^{142}$; the regional atrophy pattern is variable, and temporal, parietal, frontal and occipital lobe atrophy is common $^{142,143}$. See BOX 2 and REF. ${ }^{144}$ for more details.

Of note, DLB and PDD share many pathological and clinical features and are usually considered as two clinical entities on a spectrum of Lewy body disease ${ }^{8,11}$. From a neuropsychological perspective, it has been shown that PDD and DLB may have different cognitive profiles, such as the presence of a more severe impairment in executive functions for PDD and in memory for DLB, and trajectories of cognitive decline, which appear to be more rapid for DLB in the language domain ${ }^{145}$. However, at the earliest stages of dementia, worse performance on tests of attention and executive functions and constructive abilities has been observed in DLB than in $\mathrm{PDD}^{146}$. Traditionally, the 1-year rule has been used to distinguish DLB from PDD: if dementia occurs more than 1 year after the diagnosis of $\mathrm{PD}$, the diagnosis is $\mathrm{PDD}$, whereas parkinsonism occurring after or simultaneously with dementia is classified as DLB. Diagnostic criteria of PD that proposed to include also parkinsonism in the context of established dementia were proposed in 2015 (REF. ${ }^{13}$ ). However, here, we refer to PDD using the traditional classification, that is, dementia occurring in a person who has been diagnosed with PD (see Outlook, below).

Computerized cognitive testing. Digital computerized cognitive testing, which can be carried out remotely from patients' homes, has become an interesting alternative to traditional pen-and-paper testing ${ }^{147}$. Benefits of computerized testing include the opportunity for frequent testing with less learning effects, which increases the sensitivity to detect decline ${ }^{148}$, cost-efficiency and the 
availability of large normative databases. Opportunities for conducting both remote functional assessments and digital interventions (such as cognitive training) on the same online platform are being studied ${ }^{147,149}$.

For the MoCA, a telephone version is available without the visual elements and it can also be administered audiovisually via several media (www.mocatest.org). The Telephone Interview for Cognitive Status (TICS) has been used in several patient groups but hardly in those with $\mathrm{PD}^{150}$. In one systematic review, the MMSE, MoCA and several neuropsychological tests showed good teleneuropsychological validity compared with face-to-face testing, although the number of studies per test was limited ${ }^{151}$. However, many challenges in teleneuropsychology remain such as copyright issues, the need for publishers' permission to adjust tests for teleneuropsychology and the need for a stable internet connection. In addition, remote assessment is difficult in people with severe cognitive or motor symptoms and with hearing or visual impairment ${ }^{152}$, and not all patients have internet access or electronic devices on which to perform this. Given these limitations, face-to-face testing is routine in clinical care and more research is needed to understand how computerized testing can provide additional and more reliable information.

\section{Biomarkers of cognitive decline}

Many of the pathologies associated with cognitive impairment can be identified in vivo using a variety of imaging and blood-based or CSF-based markers. These biomarkers can be used to provide an increased understanding of the mechanisms underlying cognitive impairment in PD and, from a clinical perspective, can identify patients with an increased risk of early and rapid cognitive decline ${ }^{153}$.

One of the first identified predictive markers was temporo-parietal atrophy on MRI (which is indicative of AD pathology $)^{154}$, confirmed in many subsequent studies. In addition, basal forebrain atrophy observed using MRI is also associated with cognitive impairment in $\mathrm{PD}^{71,72}$. Hypometabolism in the medial frontal and parietal regions using FDG-PET is associated with a decline in executive and memory function ${ }^{155}$. More recent MRI techniques, such as diffusion tensor imaging, also hold promise as biomarkers of cognitive function ${ }^{156}$. For example, increased radial and axial diffusivity in the thalamus observed using diffusion tensor imaging was associated with a decline in MoCA scores ${ }^{157}$.

In addition to general imaging biomarkers, markers for specific pathologies that are associated with cognitive impairment are available. For example, CSF markers of $\mathrm{AD}$ pathology can predict future cognitive decline ${ }^{158}$. Indeed, in one study, low amyloid- $\beta_{1-42}(A \beta 42)$ levels were associated with the development of MCI or dementia ${ }^{153}$. Evidence for an association between CSF total tau or phospho-tau levels and MCI or dementia in PD has been limited mostly to cross-sectional studies ${ }^{159}$, although the potential of CSF total tau, in combination with CSF A $\beta 42$ and caudate [ ${ }^{123}$ I]FP-CIT uptake, in predicting the development of cognitive impairment has been reported ${ }^{160}$. A recent PET study did not report associations between tau pathology and cognition in $\mathrm{PD}^{161}$.
An a-synuclein biomarker for cognitive impairment may prove difficult owing to the central role of $\alpha$-synuclein in PD itself. CSF levels of total $\alpha$-synuclein have been inconsistently associated with cognitive decline, with some studies reporting reduced concentrations whereas others report increased concentrations ${ }^{162}$. Possibly, early in $\mathrm{PD}$, there is a reduced concentration of $\alpha$-synuclein, linked to $\alpha$-synuclein being included in the formation of Lewy bodies, followed by increased concentrations due to leakage of $\alpha$-synuclein associated with more neurodegeneration ${ }^{163}$. Recent studies using seed technology (a group of highly sensitive protein amplification assays used for the detection of aggregates of misfolded proteins) for strains have reported clearer associations with Lewy body pathology and might provide a more accurate predictor of cognitive decline ${ }^{164}$.

There is emerging evidence supporting the role of quantitative electroencephalography (EEG) as a diagnostic marker for DLB, with slower wave activity and variation in dominant frequency in patients with this disorder ${ }^{11,12}$. Similar changes, such as quantitative EEG background slowing-down and spectral power analysis performed with machine learning techniques, are associated with cognitive impairment in Lewy body disease ${ }^{165,166}$ and preliminary studies have suggested EEG as a predictive biomarker of cognitive decline in $\mathrm{PD}^{167}$. In a subsequent study, an increased risk of dementia in patients with PD with low background rhythm frequency and increased theta median power was found ${ }^{168}$.

\section{Management}

\section{Pharmacotherapy for dementia}

Most randomized controlled trials (RCTs) for cognition in PD have focused on patients with dementia (TABLE 2); however, as PDD together with DLB are often considered as part of a broader clinicopathological entity called Lewy body dementia, several RCTs have included both patients with PDD or DLB.

To date, the only unequivocally positive RCT for PDD was for the cholinesterase inhibitor (ChEI) rivastigmine $^{169}$. ChEIs reversibly inhibit the enzyme acetylcholinesterase, which decreases the metabolism of acetylcholine and enhances cholinergic neurotransmission in the basal forebrain. In this trial, rivastigmine had statistically significant but clinically modest effects on a range of primary (ADAS-Cog) and secondary (such as ADCS-CGIC, ADCS-ADL, verbal fluency, attention, and visuospatial abilities) outcome measures. Accordingly, oral rivastigmine is FDA-approved and EMA-approved for the treatment of mild-to-moderate PDD but not of PD-MCI due to a lack of efficacy in a single randomized placebo-controlled trial ${ }^{170}$. Both rivastigmine capsules and transdermal patches have a similar efficacy in improving cognition and behavioural symptoms albeit with greater improvements observed for the oral formulation ${ }^{171}$. In terms of tolerability, in the pivotal placebo-controlled RCT, nausea, vomiting and tremor were statistically more common in the rivastigmine capsule group compared with placebo $^{169}$. A large RCT of donepezil, another ChEI, for PDD produced an improvement in cognitive performance assessed using ADAS-Cog, although this did 
Table 2 | Published RCTs investigating treatments for mild cognitive impairment and dementia in Parkinson disease

\begin{tabular}{|c|c|c|c|c|c|}
\hline Treatment & Dose & $n$ & Duration & $\begin{array}{l}\text { Summary of primary results (active group } \\
\text { vs placebo/control) }\end{array}$ & Ref. \\
\hline \multicolumn{6}{|l|}{ Dementia } \\
\hline Donepezil & $10 \mathrm{mg} /$ day & 16 & 18 weeks & $\leftrightarrow$ global cognition, $\uparrow$ memory & 281 \\
\hline Donepezil & $10 \mathrm{mg} /$ day & 22 & 10 weeks & $\leftrightarrow$ global cognition & 282 \\
\hline Donepezil & $5 / 10 \mathrm{mg} /$ day & 550 & 24 weeks & $\begin{array}{l}\leftrightarrow \text { global cognition, } \uparrow \text { clinician’s global } \\
\text { impression of change }\end{array}$ & 172 \\
\hline Galantamine & $16 \mathrm{mg} /$ day & 41 & 24 weeks & $\begin{array}{l}\uparrow \text { global cognition, } \uparrow \text { frontal lobe function, } \\
\uparrow \text { visuospatial function }\end{array}$ & 283 \\
\hline IRL752 & $750 \mathrm{mg} /$ day & 32 & 4 weeks & $\begin{array}{l}\leftrightarrow \text { spatial working memory, } \uparrow \text { executive } \\
\text { functions (secondary outcomes) }\end{array}$ & 219 \\
\hline Memantine & $20 \mathrm{mg} /$ day & 25 & 16 weeks & $\leftrightarrow$ global cognition & 284 \\
\hline Memantine & $20 \mathrm{mg} /$ day & 40 & 24 weeks & $\uparrow$ clinician’s global impression of change & 173 \\
\hline Memantine & $20 \mathrm{mg} /$ day & 120 & 24 weeks & $\leftrightarrow$ clinician's global impression of change & 174 \\
\hline Rivastigmine & $12 \mathrm{mg} /$ day & 541 & 24 weeks & $\begin{array}{l}\uparrow \text { global cognition, } \uparrow \text { clinician’s global } \\
\text { impression of change }\end{array}$ & 169 \\
\hline \multicolumn{6}{|l|}{ Mild cognitive impairment } \\
\hline Cognitive rehabilitation therapy & 2 hours/week & 20 & 6 weeks & $\uparrow$ attention, $\leftrightarrow$ all other domain-specific tests & 285 \\
\hline Cognitive rehabilitation therapy & 135 minutes/week & 31 & 4 weeks & $\begin{array}{l}\uparrow \text { global cognition, } \uparrow \text { memory, } \uparrow \text { executive } \\
\text { functions, } \leftrightarrow \text { all other domain-specific tests }\end{array}$ & 286 \\
\hline Cognitive training therapy & $\begin{array}{l}135 \text { minutes/week plus home } \\
\text { exercises }\end{array}$ & 46 & 4 weeks & $\leftrightarrow$ global cognition & 287 \\
\hline $\begin{array}{l}\text { Cognitive training therapy plus } \\
\text { tDCS }\end{array}$ & $\begin{array}{l}120 \text { minutes/week plus } \\
80 \text { minutes/week }\end{array}$ & 24 & 4 weeks & $\begin{array}{l}\downarrow \text { attention/executive functions, } \leftrightarrow \text { all other } \\
\text { domain-specific tests }\end{array}$ & 222 \\
\hline $\begin{array}{l}\text { Standard cognitive training or } \\
\text { tailored cognitive training with } \\
\text { or without tDCS }\end{array}$ & $\begin{array}{l}\text { Cognitive training: } \\
135 \text { minutes/week; tDCS: } \\
20 \text { minutes/week }\end{array}$ & 42 & 4 weeks & $\begin{array}{l}\uparrow \text { executive function, } \uparrow \text { memory, } \uparrow \text { attention/ } \\
\text { working memory, } \uparrow \text { language, } \uparrow \text { activities of } \\
\text { daily living, } \uparrow \text { quality of life }\end{array}$ & 288 \\
\hline tDCS plus physical therapy & 25 minutes/day & 20 & 2 weeks & $\uparrow$ global cognition & 223 \\
\hline Atomoxetine & $80 \mathrm{mg} /$ daily & 30 & 10 weeks & $\leftrightarrow$ all domain-specific tests & 182 \\
\hline Creatine plus coenzyme Q10 & $10 \mathrm{~g} /$ day plus $300 \mathrm{mg} /$ day & 75 & 12-18 months & $\uparrow$ global cognition, $\uparrow$ plasma phospholipid levels & 214 \\
\hline Rasagiline & $1 \mathrm{mg} /$ day & 55 & 12 weeks & $\begin{array}{l}\uparrow \text { working memory, } \uparrow \text { verbal fluency, } \leftrightarrow \text { all other } \\
\text { domain-specific tests }\end{array}$ & 289 \\
\hline Rasagiline & $1 \mathrm{mg} /$ day & 170 & 24 weeks & $\leftrightarrow$ global cognition & 178 \\
\hline Rivastigmine & $9.5 \mathrm{mg} / 24$ hours & 28 & $\begin{array}{l}10 \text { weeks per } \\
\text { treatment phase }\end{array}$ & $\leftrightarrow$ clinician's global impression of change & 170 \\
\hline
\end{tabular}

not reach statistical significance ${ }^{172}$. No randomized, double-blind RCTs of galantamine, another ChEI, for PDD have been conducted. Although donepezil and galantamine have insufficient evidence for the treatment of PDD, they have been rated as 'possibly useful' by the International Parkinson and Movement Disorder Society Evidence-Based Medicine Committee because of their proven effects and regulatory approval for $\mathrm{AD}^{15}$.

Memantine, an NMDA receptor antagonist that reduces glutamatergic neural transmission and glutamate toxicity in the brain, is approved by the FDA and EMA for the treatment of moderate-to-severe AD. The efficacy of memantine was investigated in two RCTs for Lewy body dementia: memantine was partially beneficial in terms of global clinical status for PDD in one study ${ }^{173}$ but not in the other ${ }^{174}$. The effects of ChEIs and the inconsistent effects of memantine have been demonstrated in several meta-analyses ${ }^{175}$.

The 5-HT6 antagonist SYN120, repurposed from $\mathrm{AD}$, has also been evaluated for the treatment of cognitive impairment in PD but negative findings were reported (NCT02258152). Intepirdine, another 5-HT6 antagonist, did not show positive effects on cognition or parkinsonism in DLB (HEADWAY-DLB Study; NCT02669433). The management of psychiatric features associated with PDD, such as depression, hallucinations and other psychotic symptoms, has been extensively reviewed elsewhere ${ }^{15,176}$. 


\section{Treatment of $\mathrm{MCl}$}

No approved treatments for PD-MCI are available, but the symptomatic treatment for this indication is of great interest to the PD community. As PD-MCI is often a transitional state to PDD, treatments are urgently needed to slow its progression to PDD, either through long-term symptomatic or disease-modification effects. The RCT landscape for PD-MCI has been quite limited $^{15,177}$, with failed studies for both a PD MAO-B inhibitor, rasagiline ${ }^{178}$, and a ChEI patch, rivastigmine ${ }^{170}$, although the latter study showed a secondary benefit on a performance-based measure of cognitive functioning (TABLE 2). In a psychosis prophylaxis study including patients without dementia on the basis of MMSE score $\geq 24$, donepezil treatment was associated with better performance on the MMSE and on an auditory memory task over a period of nearly 2 years ${ }^{179}$. In addition, preliminary studies of atomoxetine, a selective noradrenaline reuptake inhibitor, showed cognitive benefit ${ }^{180,181}$, yet a subsequent small RCT in PD-MCI did not find a benefit on cognitive tests despite significant improvements in subjective reporting ${ }^{182}$. Ongoing or planned studies for PD-MCI include a selective $\alpha 7$ nicotinic acetylcholine receptor agonist and multiple non-pharmacological treatments.

\section{Non-pharmacological approaches}

Non-pharmacological therapies for cognition in PD fall into four broad categories: cognitive interventions (such as engagement in cognitive and social activities, guided practice on tasks or mnemonic strategies, and individualized treatment plans that focus on compensatory strategies), physical exercise (such as treadmill training), non-invasive brain stimulation (either transcranial direct current stimulation (tDCS) or repetitive transcranial magnetic stimulation (rTMS)), and invasive brain stimulation (DBS) (TABLE 2). Although the sophistication of studies has improved over time, many studies have numerous severe methodological limitations such as small sample sizes and lack of application of diagnostic criteria for PD-MCI or PDD ${ }^{183}$. Another important limitation is the difficultly in conducting double-blind studies, thereby introducing the high likelihood of non-specific treatment effects for patients randomized to the active treatment arm; additionally, even in double-blind studies, the effectiveness of the blinding process is rarely reported.

Despite the study limitations, there is preliminary evidence from reviews or quantitative meta-analyses, albeit with mixed findings based on limited data of varying quality ${ }^{184}$, that cognitive training ${ }^{185,186}$, physical exercise $^{187,188}$ and non-invasive brain stimulation ${ }^{189}$ may all lead to at least short-term benefit in some cognitive abilities, with the strongest evidence for executive function abilities. In terms of cognitive training, one systematic review found that the use of multi-domain, computer-based cognitive training at a frequency of 2-3 times per week over 3-12 weeks is associated with measurable improvements in executive functions, memory, processing speed and attention ${ }^{190}$. However, another systematic review and meta-analysis graded the evidence from published clinical trials on cognitive training as low and recommended further large-scale studies in $\mathrm{PD}^{186}$. Regarding exercise, some studies have suggested that aerobic exercise, among other types of physical exercise, provides specific benefits for memory, although studies vary widely in the amount of exercise studied (between 30 and 60 minutes per session, 1-3 times per week, for 4-26 weeks) ${ }^{191}$. In particular, aerobic and resistance exercise (such as treadmill training), and combined physical and cognitive training, have shown short-term maintainance/improvement of global cognition, processing speed, sustained attention, mental flexibility and memory in patients with $\mathrm{PD}^{188}$.

In terms of the neural stimulation techniques that have been evaluated in PD, tDCS modulates neural activity by delivering low-intensity electrical currents to specific cortical regions ${ }^{192}$, whereas rTMS induces an electrical field in the brain by using a magnetic field, thus leading to neuronal depolarization ${ }^{193}$. There is insufficient RCT evidence to recommend tDCS or rTMS for the treatment of cognitive impairment in $\mathrm{PD}^{15}$. For DBS, one small study used a sham-controlled, crossover, bilateral DBS of the nucleus basalis of Meynert in PDD and showed that the procedure was safe; however, the primary cognitive outcomes did not significantly improve, although there was evidence for improvement in neuropsychiatric symptoms with DBS ${ }^{194}$.

\section{Impact of PD treatments}

The clinical choice of initial PD medication (levodopa, dopamine agonist or monoamine oxidase-B (MAO-B) inhibitor) at disease onset does not seem to make a difference in terms of cumulative dementia rates ${ }^{195,196}$. However, there is strong evidence that medications with anticholinergic properties (encompassing both PD anticholinergic medications, such as benztropine and trihexyphenidyl, and over-the-counter sleep medications or antihistamines, such as diphenhydramine), and particularly the long-term exposure to multiple medications or medications with greater anticholinergic properties, are associated with worse long-term cognition in the general population and patients with $\mathrm{PD}^{197,198}$, and thus represent a target for clinical management ${ }^{199}$. In patients with PDD, simplification of antiparkinsonian treatment through a stepwise withdrawal of non-levodopa PD medications starting with anticholinergic drugs, followed by amantadine, selegiline, dopamine agonists and then catechol-O-methyltransferase inhibitors, might be useful, particularly if comorbid psychosis is present ${ }^{176}$.

In addition, several studies have found that DBS can worsen cognitive functioning ${ }^{200}$; as a result, cognitive testing is recommended as part of the pre-DBS surgery evaluation process, and patients with severe cognitive impairment should not undergo brain surgery. However, the use of model-based stimulation parameters to minimize the spread of the electrical current to non-motor portions of the subthalamic nucleus reversed the cognitive decline that occurred after DBS in one study ${ }^{201}$. Encouragingly, a subsequent study of DBS in younger patients with shorter disease duration showed short-term cognitive tolerability similar to the best medical therapy ${ }^{202}$. 
Other device-aided PD treatments, such as continuous subcutaneous apomorphine infusion and intrajejunal levodopa infusion (IJLI), despite being avoided in those with PD-associated cognitive impairment, are now considered as potential therapeutic strategies even in patients with MCI (apomorphine and IJLI) and mild-to-moderate dementia (IJLI) ${ }^{203,204}$. Patients with cognitive complaints as part of non-motor fluctuations ${ }^{205,206}$ could potentially benefit cognitively from adjustments to their PD treatments, although this remains to be demonstrated.

\section{Indirect management strategies}

Given the association between common non-motor symptoms, such as depression and RBD, and cognitive decline in $\mathrm{PD}$, it is possible that treating these disorders may affect cognitive abilities in the short or long term, although this has not yet been demonstrated. Given the known associations between vascular risk factors ${ }^{207,208}$ and pathology $y^{209}$, orthostatic hypotension ${ }^{210}$, obstructive sleep apnoea ${ }^{211,212}$, excessive daytime sleepiness ${ }^{213}$ and cognitive performance in PD is important in this regard. Indirect management strategies for cognitive impairment are based on treating the comorbid disorders and risk factors; for example, managing the comorbid disorders associated with cognitive impairment (such as depression, psychosis and RBD) and managing comorbid vascular disease and vascular risk factors (such as hypertension, diabetes mellitus and dyslipidemia) given the association between cognitive impairment and vascular pathology in $\mathrm{PD}^{209}$. Specifically, obstructive sleep apnoea should be treated using continuous positive airway pressure ventilation and symptomatic orthostatic hypotension should be treated with midodrine, fludrocortisone or droxidopa, given their association with impaired cognition in patients with $\mathrm{PD}^{210,212}$. In addition, another indirect management strategy is minimizing anticholinergic medication use, including using instruments such as the Anticholinergic Cognitive Burden Scale ${ }^{198}$ to identify and rate anticholinergic medications.

\section{Novel treatment approaches}

In general, disease-modifying clinical trials for PD do not determine if patients meet diagnostic criteria for a cognitive disorder or assess cognitive performance or its change over time.

To date, there has been one completed neuroprotective RCT for cognitive function in PD - a study testing the combination of the purported neuroprotectants creatine and coenzyme Q10 (CoQ10) ${ }^{214}$. CoQ10 plays an important role in mitochondrial bioenergetics, protects the integrity of biological membranes, and acts as an intracellular antioxidant and free-radical scavenger ${ }^{215}$, and creatine, an endogenous organic acid, is also an active component of mitochondrial metabolism and has antioxidant properties ${ }^{216}$. This 18-month study randomized patients with PD-MCI to either monohydrate creatine plus CoQ10 or placebo, and both cognitive function (assessed using the MoCA) and a treatment-related biological measure (plasma phospholipid level, a measure of cell membrane integrity) showed improvements in the treatment group compared with placebo. Although these results are promising, there was no mention of discontinuations, adverse events or other neuropsychological measures, and other studies of both compounds in PD were negative or did not provide enough evidence for their neuroprotective effects ${ }^{217,218}$.

Other ongoing or recently completed studies for PDD with novel therapeutic approaches include testing a partial D1 positive allosteric modulator (NCT03305809), an antibiotic (ceftriaxone, NCT03413384), a pharmacological chaperone for glucocerebrosidase (ambroxol, NCT02914366), human plasma fractions (NCT03713957), an NMDAR modulator (NCT04148391), a cortical enhancer ${ }^{219}$ and a sigma 1 receptor agonist ${ }^{220}$. The latter, in particular, was evaluated in a double-blind, multicenter, placebo-controlled phase II trial and showed positive results for multiple subtests of the Cognitive Drug Research computerized assessment system for the active group versus placebo ${ }^{220}$. However, these encouraging preliminary data need further validation in a larger RCT. For the related disorder of DLB, one completed phase II double-blind, placebo-controlled RCT found that the oral p38a kinase inhibitor neflamapimod significantly improved cognition on a hybrid (computerized and paper-and-pencil) neuropsychological battery ${ }^{221}$, although conclusions on its efficacy and possible use in clinical practice will require positive results in a phase III clinical trial.

Given the multifactorial aetiology of cognitive impairment in $\mathrm{PD}$, it is unlikely that one single treatment strategy is sufficient and combinations, for example, of pharmacological and non-pharmacological therapies, are likely to be more successful in managing and preventing cognitive decline. We are not aware of such studies but, for instance, studies combining cognitive training or physical therapy with tDCS do exist ${ }^{222,223}$. Combination therapies should therefore be further tested.

\section{Quality of life}

In addition to an association with increased mortality ${ }^{224,225}$ and complicating the management of motor symptoms, the presence of cognitive impairment plays an important role in determining the health-related QOL (HRQOL) in people with PD. HRQOL in patients with PD is a pillar of assessment of health empowering the patient, with a crucial role in defining individual well-being and global health ${ }^{226,227}$. Validated tools for the assessment of HRQOL include the Parkinson's Disease Questionnaire-39 (PDQ-39) 228 , the Parkinson's Disease Questionnaire-8 (PDQ-8) ${ }^{229}$ and the European Quality of Life - Five Dimensions (EQ-5D) $)^{230}$. Caregiver stress can be evaluated by, for example, the Zarit Burden Interview ${ }^{231,232}$. However, of note, these tools address the cognitive related-aspects of HRQOL only indirectly through, for example, assessment of the experienced impairment in activities of daily living.

A number of factors contribute to reduced HRQOL in PD (FIG. 5). The burden of non-motor symptoms drives HRQOL, as demonstrated by a multicenter, international, cross-sectional study on 411 patients with $\mathrm{PD}$ that found that non-motor symptoms, including cognitive impairment, have, as a whole, a greater effect 


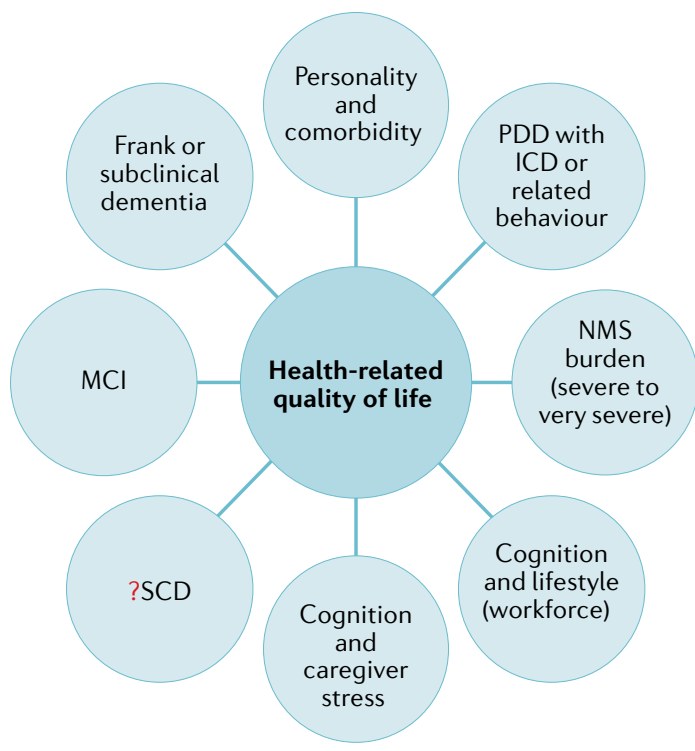

Fig. 5 | Determinants of quality of life associated with cognitive impairment in PD. The different cognitive syndromes associated with Parkinson disease (PD), PD dementia (PDD), mild cognitive impairment (MCI) or subjective cognitive decline (SCD) directly impact the health-related quality of life. In addition, an indirect effect of cognitive impairment on health-related quality of life can be exerted through their impact on other determinants of quality of life such as caregiver stress, comorbidities and overall non-motor symptom (NMS) burden. ICD, impulse control disorder.

on HRQOL than motor symptoms and that progression of non-motor symptoms contributes to HRQOL decline $^{226}$. The authors of this study suggested that these findings might be explained by the fact that the presence of dopaminergic therapy and, therefore, the impact of the motor manifestations on HRQOL, may be neutralized by effective antiparkinsonian treatment ${ }^{226}$. In addition, only a minority of non-motor symptoms, due to their mainly non-dopaminergic nature, respond to dopaminergic therapy and this, together with a range of barriers in reporting non-motor symptoms among patients and clinicians ${ }^{233}$, might prevent their effective management ${ }^{226}$.

The HRQOL of patients with PD and cognitive impairment, and specifically regarding attention and memory deficits as assessed by the NMSS, is significantly worse compared to that of patients without these impairments ${ }^{226}$. The ICICLE cohort study showed that even PD-MCI leads to poorer QOL over 3 years of follow-up and specifically in those who developed dementia during follow-up ${ }^{234}$. In addition to global cognition, impaired attention was a particularly strong determinant of QOL, demonstrated by multivariate modelling showing that attentional deficits had the strongest predictive power ${ }^{234}$.

Equally important is the impact of cognitive impairment on the caregiver. Both cognitive impairment ${ }^{235}$ and other PD-related non-motor symptoms that are associated with PDD, including psychosis, apathy, depression and impulsive control disorders ${ }^{236-239}$, contribute to the burden of caring for people with PD. For example, in one study including 584 pairs of patients with PD and their primary caregivers, the cumulative burden of neuropsychiatric symptoms coupled with dementia appeared to be a major determinant of QOL ${ }^{240}$. The perceived burden of care is closely linked to the positive quality of the relationship between the patient and the caregiver (mutuality). Indeed, mutuality is negatively influenced by cognitive impairment and, in turn, this effect on mutuality negatively affects the perceived burden of care ${ }^{241}$. In addition, cognitive impairment in patients with PDD significantly contributes to poorer mental health, stress, negative strain, resentment and overall higher levels of care burden in patients' spouses and life partners, who constitute the majority of caregivers ${ }^{242,243}$.

Thus, HRQOL assessment and focus on the partner and the patient-carer relationship should be integral to any cognitive assessment, and specific personalized aspects need to be considered in people with PD and cognitive impairment.

HRQOL has now emerged as a key issue in the emergence of the long COVID/post-acute COVID-19 syndrome in patients with $\mathrm{PD}$, and a new report suggests that cognitive impairment may play a key part in the symptoms that constitute long COVID in $\mathrm{PD}^{244}$. The overall effect of this phenomenon needs to be ascertained in longitudinal studies on patients affected by COVID-19 and some such studies have already started.

\section{Outlook}

Global burden of PD-associated cognitive impairment $\mathrm{PD}$ is the fastest growing neurological disorder in the world in terms of prevalence, disability and deaths ${ }^{245}$. In 2016, it has been estimated that 6.1 million individuals had PD globally, compared with 2.5 million in 1990 , and this number is expected to more than double by 2040 (REF. ${ }^{246}$ ). In light of what has been defined as the "Parkinson pandemic" ${ }^{247}$, more attention has been focused in recent years on the impact of PD in lowermiddle-income and low-income countries, where the largest increases in prevalence are expected ${ }^{248-250}$. On the other hand, the global number of individuals who lived with dementia has been estimated to be 43.8 million in 2016, expected to increase to over 100 million by $2050\left(\mathrm{REF}^{251}\right)$. However, while care inequalities in dementia care across the globe $\mathrm{e}^{252,253}$ and research challenges in developing countries are increasingly being recognized for both PD and dementia separately ${ }^{254,255}$, data on the prevalence of $\mathrm{PD}$-associated cognitive impairment, risk prediction, management and societal burden in these regions are lacking. Addressing these disparities with strategies to increase access to health care, research funding and public awareness on the topic is therefore mandatory and represents a global health priority.

\section{Classification issues and prodromal stages}

The proposal that dementia prior to or simultaneous with motor symptoms can be included in the diagnostic criteria for $\mathrm{PD}^{13,256}$ has reopened the long-standing debate on whether PDD and DLB should be considered the same disease ${ }^{257-260}$. A deeper understanding of the pathophysiological processes underlying these two synucleinopathies, such as the relative contribution of 
$\beta$-amyloid and tau pathology in cortex and striatum, the extent of cortical Lewy pathology and $\alpha$-synuclein load in the hippocampus, the severity of neuronal loss in the substantia nigra, and cholinergic cell loss ${ }^{260}$, is required to better understand the relationship between PD and DLB.

Although some risk factors for cognitive impairment have been identified ${ }^{46,48,261}$, further research is needed to better identify any early evidence of cognitive impairment in genetic at-risk populations and in individuals with clinical features of prodromal PD to provide opportunities for prevention strategies and early precision therapy interventions.

\section{Predictive biomarkers}

Studies have identified a specific brain-clinical pattern that identifies people with RBD who developed rapid cognitive decline and DLB rather than PD. Based on routine MRI using partial least squares, atrophy in the basal ganglia, thalamus, amygdala, and frontotemporal grey and white matter as well as the expansion of CSF-filled spaces predicted cognitive decline in both RBD and $\mathrm{PD}^{262}$. In addition to imaging, CSF and EEG biomarkers for cognitive impairment, there is an increasing focus on exploring $\alpha$-synuclein and other biomarkers in other biofluids and tissues such as skin, colon, submandibular gland, CSF, saliva and blood ${ }^{263}$.

In this scenario, the development of plasma-based biomarkers for cognitive impairment in PD is particularly relevant given the recent progresses made in $\mathrm{AD}$ and the promise of neurofilament light and other plasma-based markers ${ }^{264}$. However, only one study has found significantly higher plasma total $\alpha$-synuclein concentrations in people with PD, in particular in those with a more advanced disease stage and dementia ${ }^{265}$. Further longitudinal studies are needed to test the hypothesis that plasma $\alpha$-synuclein could predict future cognitive decline in PD. Seed technology techniques using protein amplification assays, such as the Protein-Misfolding Cyclic Amplification (PMCA) and the Real-Time Quaking-Induced Conversion (RT-QuIC), are able to detect synucleinopathies with very high sensitivity and specificity even at the preclinical stage ${ }^{164}$, although their potential use in the prediction of cognitive decline in PD needs to be explored. Another unmet need in biomarker development is represented by the lack of reliable a-synuclein PET ligands, which will allow the determination of the in vivo distribution of Lewy body pathology. Other novel imaging techniques also have huge potential to detect the earliest brain changes leading to cognitive impairment in $\mathrm{PD}^{156}$.

\section{The era of digital cognitive testing}

The development of digital cognitive testing and the evolution of self-completed computerized assessments and wearable devices to assess cognitive functioning in daily life ${ }^{147,149}$ provides an exciting opportunity to both improve clinical management and to obtain more sensitive outcome measures for clinical trials and will likely become a standard procedure in the future, given further technological improvements and increased access to the internet and digital devices. To reach this point, psychometric requirements (reliability, validity and normative data), documentation and technical problems, as well as their relation to traditional tests, need to be well known ${ }^{266}$.

\section{Management}

Several questions on the direct and indirect management of cognitive symptoms in PD remain open.

Important challenges concern the role and long-term validity of non-pharmacological interventions, such as cognitive training, exercise-based therapy and noninvasive brain stimulation, in addressing and preventing cognitive dysfunction in PD. So far, clinical trials focused on these strategies, despite showing encouraging results, have been hindered by methodological issues, poor assessment of long-term effects and scarcity of pathophysiological correlates. In future trials, a more robust study design ${ }^{186}$, longer intervention and follow-up durations as well as in vivo pathophysiological evidence (such as that provided by neuroimaging) will be the key components to establish the true role of such therapies.

\section{The need for disease-modifying therapies}

Numerous disease-modifying compounds targeting multiple pathophysiological processes are being tested in $\mathrm{PD}$, although the process of bringing them into clinical use in PD remains a long-standing challenge ${ }^{267}$.

Successful disease-modifying drugs for PD should also have cognitive benefit, although cognition has rarely been included in these studies. For instance, preclinical models suggest that immunotherapies targeting both $\beta$-amyloid and $\alpha$-synuclein reduce $A D$ and $P D$ pathological burden, improve behaviours and may have an additive effect ${ }^{268}$. Active and passive immunotherapies targeting multiple pathologies, alone or in combination, therefore represent one of the most intriguing opportunities to tackle cognitive impairment in $\mathrm{PD}^{269-271}$.

Diabetes-related pathways seem to play a role in the pathogenesis of $\mathrm{PD}$, potentially through peripheral and cerebral insulin resistance leading to altered autophagy, mitochondrial function, cell proliferation and increased inflammation, which may have positive effects on memory and cognition ${ }^{272}$. The disease-modifying and neuroprotective potential in PD of antidiabetic agents is currently being explored in several trials $\mathrm{s}^{272}$. Additional repurposed candidates include angiotensin receptor and calcium-channel blockers, tyrosine kinase inhibitors, immunomodulators, and GBA-related agents including ambroxol and anti-oxidants ${ }^{273}$. Most studies have been negative but still provide important lessons to learn, both regarding the most promising targets as well as trial designs.

\section{Patient and public involvement}

In the past years, there has been growing attention on the need to include patients, their caregivers and families in all stages of the research process ${ }^{274}$. The increasing contribution of patient and public involvement groups in defining research questions, designing and conducting clinical trials, disseminating outcomes, and shaping research roadmaps reflects the concept of research as a shared effort among all stakeholders. Although in PD research this concept is increasingly 
being recognized ${ }^{275}$, further involvement of patients and families, also inclusive of diverse patient populations, in research focused on $\mathrm{PD}$-associated cognitive impairment is needed.

\section{Improved clinical trial design}

Clinical trials for therapies targeting cognition in PD may benefit from recent design improvements. More sensitive outcomes, including computerized cognitive testing and wearables to measure motor and other functions, together with the development of an internationally recognized set of core outcomes, as has been done for idiopathic $\mathrm{PD}^{276}$, particularly focused on patients with cognitive impairment and on the effects of specific interventions (such as non-pharmacological interventions), will allow the reporting and comparison of research outcomes in a standardized manner. More targeted selection criteria using current diagnostic criteria ${ }^{7,8}$ and recommended assessments ${ }^{120}$, combined with both biomarkers and genetic risk factors aiming to assign the right person to the right intervention at an early disease stage, as well as biomarkers demonstrating target involvement, will offer opportunities for improved statistical power and cheaper trials.

Published online: 01 July 2021
1. Zaman, V et al. Cellular and molecular pathophysiology in the progression of Parkinson's disease. Metab. Brain Dis. 36, 815-827 (2021).

2. Aarsland, D et al Risk of dementia in Parkinson's disease: a community-based, prospective study. Neurology 56, 730-736 (2001).

3. Leroi, I., McDonald, K., Pantula, H. \& Harbishettar, V. Cognitive impairment in Parkinson disease: impact on quality of life, disability, and caregiver burden. J. Geriatr. Psychiatry Neurol. 25, 208-214 (2012).

4. Vossius, C., Larsen, J. P., Janvin, C. \& Aarsland, D. The economic impact of cognitive impairment in Parkinson's disease. Mov. Disord. 26, 1541-1544 (2011).

5. Chandler, J. et al. Characteristics of Parkinson's disease in patients with and without cognitive impairment. J. Parkinsons Dis. https://doi.org/10.3233/ jpd-202190 (2021)

6. Jessen, F. et al. A conceptual framework for research on subjective cognitive decline in preclinical Alzheimer's disease. Alzheimers Dement. 10, 844-852 (2014)

7. Litvan, I. et al. Diagnostic criteria for mild cognitive impairment in Parkinson's disease: Movement Disorder Society Task Force guidelines. Mov. Disord. 27, 349-356 (2012)

MDS PD-MCI criteria making the construct more concrete. These clinical criteria have led to more coherence in the PD field.

8. Emre, M. et al. Clinical diagnostic criteria for dementia associated with Parkinson's disease. Mov. Disord. 22 1689-1707 (2007). quiz 1837.

9. Goetz, C. G., Emre, M. \& Dubois, B. Parkinson's disease dementia: definitions, guidelines, and research perspectives in diagnosis. Ann. Neurol. 64, S81-S92 (2008).

10. Harvey, P. D. Domains of cognition and their assessment. Dialogues Clin. Neurosci. 21, 227-237 (2019).

11. McKeith, I. G. et al. Diagnosis and management of dementia with Lewy bodies: fourth consensus report of the DLB Consortium. Neurology 89, 88-100 (2017).

12. McKeith, I. G. et al. Research criteria for the diagnosis of prodromal dementia with Lewy bodies. Neurology 94, 743-755 (2020).

13. Postuma, R. B. et al. MDS clinical diagnostic criteria for Parkinson's disease. Mov. Disord. 30, 1591-1601 (2015).

14. Goldman, J. G. \& Weintraub, D. Advances in the treatment of cognitive impairment in Parkinson's disease. Mov. Disord. 30, 1471-1489 (2015).

15. Seppi, K. et al. Update on treatments for nonmotor symptoms of Parkinson's disease-an evidencebased medicine review. Mov. Disord. 34, 180-198 (2019).

16. Fengler, $\mathrm{S}$. et al. Cognitive changes in prodromal Parkinson's disease: a review. Mov. Disord. 32 , 1655-1666 (2017)

17. Perez, F. et al. Risk of dementia in an elderly population of Parkinson's disease patients: a 15-year population-based study. Alzheimers Dement. 8 , 463-469 (2012)

18. Aarsland, D., Zaccai, J. \& Brayne, C. A systematic review of prevalence studies of dementia in Parkinson's disease. Mov. Disord. 20, 1255-1263 (2005).

19. Williams-Gray, C. H. et al. The distinct cognitive syndromes of Parkinson's disease: 5 year follow-up of the CamPalGN cohort. Brain 132, 2958-2969 (2009).
20. Williams-Gray, C. H. et al. The CamPaIGN study of Parkinson's disease: 10-year outlook in an incident population-based cohort. J. Neurol. Neurosurg. Psychiatry 84, 1258-1264 (2013).

21. Hely, M. A., Reid, W. G., Adena, M. A., Halliday, G. M \& Morris, J. G. The Sydney multicenter study of Parkinson's disease: the inevitability of dementia at 20 years. Mov. Disord. 23, 837-844 (2008). The first longitudinal study of a de novo PD cohort demonstrating the high risk of dementia over time.

22. Buter, T. C. et al. Dementia and survival in Parkinson disease: a 12-year population study. Neurology 70, 1017-1022 (2008)

23. Prince, M. et al. The global prevalence of dementia: a systematic review and metaanalysis. Alzheimers Dement. 9, 63-75 e62 (2013).

24. Aarsland, D. et al. The rate of cognitive decline in Parkinson disease. Arch. Neurol. 61, 1906-1911 (2004).

25. Aarsland, D., Larsen, J. P., Tandberg, E. \& Laake, K Predictors of nursing home placement in Parkinson's disease: a population-based, prospective study. J. Am. Geriatr. Soc. 48, 938-942 (2000).

26. Aarsland, D. et al. Mild cognitive impairment in Parkinson disease: a multicenter pooled analysis Neurology 75, 1062-1069 (2010).

27. Pedersen, K. F., Larsen, J. P., Tysnes, O. B. \& Alves, G. Natural course of mild cognitive impairment in Parkinson disease: a 5-year population-based study. Neurology 88, 767-774 (2017).

One of the first studies based on an incidence cohort, reporting the natural course of PD-MCI, diagnosed using standardized criteria over time.

28. Lawson, R. A. et al. Stability of mild cognitive impairment in newly diagnosed Parkinson's disease. J. Neurol. Neurosurg. Psychiatry 88, 648-652 (2017).

29. Domellof, M. E., Ekman, U., Forsgren, L. \& Elgh, E. Cognitive function in the early phase of Parkinson's disease, a five-year follow-up. Acta Neurol. Scand. 132, 79-88 (2015).

30. Weintraub, D. et al. Cognitive performance and neuropsychiatric symptoms in early, untreated Parkinson's disease. Mov. Disord. 30, 919-927 (2015).

31. Roberts, R. \& Knopman, D. S. Classification and epidemiology of MCl. Clin. Geriatr. Med. 29, 753-772 (2013)

32. Jones, J. D., Kuhn, T. P. \& Szymkowicz, S. M. Reverters from PD-MCl to cognitively intact are at risk for future cognitive impairment: analysis of the PPMI cohort. Parkinsonism Relat. Disord. 47, 3-7 (2018).

33. Wood, K. L. et al. Different PD-MCl criteria and risk of dementia in Parkinson's disease: 4-year longitudinal study. NPJ Parkinsons Dis. 2, 15027 (2016).

34. Erro, R. et al. Do subjective memory complaints herald the onset of mild cognitive impairment in Parkinson disease. J. Geriatr. Psychiatry Neurol. 27, 276-281 (2014).

One of the first studies demonstrating the prognostic impact of subjective memory complaints in PD.

35. Purri, R. et al. Subjective cognitive complaint in Parkinson's disease patients with normal cognition: canary in the coal mine? Mov. Disord. 35 , 1618-1625 (2020).

36. Chua, C. Y. et al. Subjective cognitive complaints in early Parkinson's disease patients with normal cognition are associated with affective symptoms Parkinsonism Relat. Disord. 82, 24-28 (2021).
37. Phongpreecha, T. et al. Multivariate prediction of dementia in Parkinson's disease. NPJ Parkinsons Dis. 6, 20 (2020).

38. Liu, G. et al. Prediction of cognition in Parkinson's disease with a clinical-genetic score: a longitudinal analysis of nine cohorts. Lancet Neurol. 16, 620-629 (2017).

39. Goetz, C. G. et al. Movement Disorder Society Task Force report on the Hoehn and Yahr staging scale: status and recommendations. Mov. Disord. 19 1020-1028 (2004).

40. Marinus, J., Zhu, K., Marras, C., Aarsland, D. $\delta$ van Hilten, J. J. Risk factors for non-motor symptoms in Parkinson's disease. Lancet Neurol. 17, 559-568 (2018).

41. Williams-Gray, C. H., Foltynie, T., Brayne, C. E., Robbins, T. W. \& Barker, R. A. Evolution of cognitive dysfunction in an incident Parkinson's disease cohort. Brain 130, 1787-1798 (2007).

42. Lee, J. E. et al. Exploratory analysis of neuropsychological and neuroanatomical correlates of progressive mild cognitive impairment in Parkinson's disease. J. Neurol. Neurosurg. Psychiatry 85, 7-16 (2014)

43. Chung, $\mathrm{S}$. J. et al. Frontal atrophy as a marker for dementia conversion in Parkinson's disease with mild cognitive impairment. Hum. Brain Mapp. 40, 3784-3794 (2019).

44. Chung, S. J. et al. Factor analysis-derived cognitive profile predicting early dementia conversion in $\mathrm{PD}$. Neurology 95, e1650-e1659 (2020)

45. Livingston, G. et al. Dementia prevention, intervention, and care: 2020 report of the Lancet Commission. Lancet 396, 413-446 (2020).

46. Chahine, L. M. et al. Cognition in individuals at risk for Parkinson's: Parkinson associated risk syndrome (PARS) study findings. Mov. Disord. 31, 86-94 (2016).

47. Gagnon, J. F. et al. Mild cognitive impairment in rapid eye movement sleep behavior disorder and Parkinson's disease. Ann. Neurol. 66, 39-47 (2009).

48. Gagnon, J. F., Bertrand, J. A. \& Genier Marchand, D. Cognition in rapid eye movement sleep behavior disorder. Front. Neurol. 3, 82 (2012).

49. Heinzel, S. et al. Update of the MDS research criteria for prodromal Parkinson's disease. Mov. Disord. 34 1464-1470 (2019).

50. Poewe, W. et al. Parkinson disease. Nat. Rev. Dis. Prim 3, 17013 (2017)

51. Bridi, J. C. \& Hirth, F. Mechanisms of $a$-synuclein induced synaptopathy in Parkinson's disease. Front. Neurosci. 12, 80 (2018).

52. Sasikumar, S. \& Strafella, A. P. Imaging mild cognitive impairment and dementia in Parkinson's disease. Front. Neurol. 11, 47 (2020).

53. Ranganath, A. \& Jacob, S. N. Doping the mind: dopaminergic modulation of prefrontal cortical cognition. Neuroscientist 22, 593-603 (2016)

54. Westbrook, A. et al. Dopamine promotes cognitive effort by biasing the benefits versus costs of cognitive work. Science 367, 1362-1366 (2020).

55. Keren, N. I. et al. Histologic validation of locus coeruleus MRI contrast in post-mortem tissue. Neuroimage 113, 235-245 (2015).

56. Borodovitsyna, O., Flamini, M. \& Chandler, D. Noradrenergic modulation of cognition in health and disease. Neural Plast. 2017, 6031478 (2017)

57. Li, Y. et al. Mild cognitive impairment in de novo Parkinson's disease: a neuromelanin MRI study in locus coeruleus. Mov. Disord. 34, 884-892 (2019). 
58. Ehrminger, M. et al. The coeruleus/subcoeruleus complex in idiopathic rapid eye movement sleep behaviour disorder. Brain 139, 1180-1188 (2016).

59. Sommerauer, M. et al. Evaluation of the noradrenergic system in Parkinson's disease: an ${ }^{11} \mathrm{C}-M e N E R$ PET and neuromelanin MRI study. Brain 141, 496-504 (2018).

60. Longardner, K., Bayram, E. \& Litvan, I. Orthostatic hypotension is associated with cognitive decline in Parkinson disease. Front. Neurol. 11, 897 (2020)

61. McDonald, C., Newton, J. L. \& Burn, D. J. Orthostatic hypotension and cognitive impairment in Parkinson's disease: causation or association? Mov. Disord. 31 , 937-946 (2016).

62. Sinn, D. I. \& Gibbons, C. H. Pathophysiology and treatment of orthostatic hypotension in parkinsonian disorders. Curr. Treat. Options Neurol. 18, 28 (2016).

63. Zhan, Y. Raza, M. U., Yuan, L. \& Zhu, M. Y Critical role of oxidatively damaged DNA in selective noradrenergic vulnerability. Neuroscience $\mathbf{4 2 2}$, 184-201 (2019)

64. van der Zee, S. et al. Monoaminergic markers across the cognitive spectrum of Lewy body disease. J. Parkinsons Dis. 8, 71-84 (2018).

65. Buddhala, C. et al. Dopaminergic, serotonergic, and noradrenergic deficits in Parkinson disease. Ann. Clin. Transl. Neurol. 2, 949-959 (2015).

66. Betts, M. J. et al. Locus coeruleus imaging as a biomarker for noradrenergic dysfunction in neurodegenerative diseases. Brain 142, 2558-2571 (2019).

67. Ballinger, E. C., Ananth, M., Talmage, D. A. \& Role, L. W. Basal forebrain cholinergic circuits and signaling in cognition and cognitive decline. Neuron 91, 1199-1218 (2016)

68. Pillet, L. E. et al. Correlation between cognition and plasma noradrenaline level in Alzheimer's disease: a potential new blood marker of disease evolution. Transl. Psychiatry 10, 213 (2020)

69. Schulz, J., Pagano, G., Fernandez Bonfante, J. A., Wilson, H. \& Politis, M. Nucleus basalis of Meynert degeneration precedes and predicts cognitive impairment in Parkinson's disease. Brain 141 1501-1516 (2018)

70. Bohnen, N. I. et al. Frequency of cholinergic and caudate nucleus dopaminergic deficits across the predemented cognitive spectrum of Parkinson disease and evidence of interaction effects. JAMA Neurol. 72 , 194-200 (2015)

71. Pereira, J. B. et al. Longitudinal degeneration of the basal forebrain predicts subsequent dementia in Parkinson's disease. Neurobiol. Dis. 139, 104831 (2020).

72. Ray, N. J. et al. In vivo cholinergic basal forebrain atrophy predicts cognitive decline in de novo Parkinson's disease. Brain 141, 165-176 (2018).

73. Alexandris, A. S. et al. Cholinergic deficits and galaninergic hyperinnervation of the nucleus basalis of Meynert in Alzheimer's disease and Lewy body disorders. Neuropathol. Appl. Neurobiol. 46 , 264-278 (2020)

74. Mattila, P. M. et al. Choline acetytransferase activity and striatal dopamine receptors in Parkinson's disease in relation to cognitive impairment. Acta Neuropathol. 102, 160-166 (2001).

75. Gaykema, R. P. \& Zaborszky, L. Direct catecholaminergic cholinergic interactions in the basal forebrain. Il. Substantia nigra-ventral tegmental area projections to cholinergic neurons. J. Comp. Neurol. 374, 555-577 (1996).

76. Gargouri, F. et al. Multimodal magnetic resonance imaging investigation of basal forebrain damage and cognitive deficits in Parkinson's disease. Mov. Disord. 34, 516-525 (2019).

77. Hall, H. et al. Hippocampal Lewy pathology and cholinergic dysfunction are associated with dementia in Parkinson's disease. Brain 137, 2493-2508 (2014).

78. Liu, A. K. L. et al. Hippocampal CA2 Lewy pathology is associated with cholinergic degeneration in Parkinson's disease with cognitive decline. Acta Neuropathol. Commun. 7, 61 (2019).

79. Nicastro, N., Garibotto, V. \& Burkhard, P. R. Extrastriatal ${ }^{123}$ I-FP-CIT SPECT impairment in Parkinson's disease - the PPMI cohort. BMC Neurol. 20, 192 (2020).

80. Pagano, G., Niccolini, F., Fusar-Poli, P. \& Politis, M. Serotonin transporter in Parkinson's disease: a meta-analysis of positron emission tomography studies. Ann. Neurol. 81, 171-180 (2017).

81. Qamhawi, Z. et al. Clinical correlates of raphe serotonergic dysfunction in early Parkinson's disease. Brain 138, 2964-2973 (2015).
82. Maillet, A. et al. The prominent role of serotonergic degeneration in apathy, anxiety and depression in de novo Parkinson's disease. Brain 139, 2486-2502 (2016).

83. Kotagal, V., Spino, C., Bohnen, N. I., Koeppe, R. \& Albin, R. L. Serotonin, beta-amyloid, and cognition in Parkinson disease. Ann. Neurol 83, 994-1002 (2018).

84. Smith, C. R. et al. Cognitive impairment in Parkinson's disease is multifactorial: a neuropsychological study. Acta Neurol. Scand. 141, 500-508 (2020).

85. Amin, J et al Neuroinflammation in dementia with Lewy bodies: a human post-mortem study. Transl. Psychiatry 10, 267 (2020).

86. Smith, C. et al. Neuropathology of dementia in patients with Parkinson's disease: a systematic review of autopsy studies. J. Neurol. Neurosurg. Psychiatry 90, 1234-1243 (2019). Assessment of neuropathology underlying dementia in $>2,000$ end-stage autopsy case showing that significant $\alpha$-synuclein pathology is the main substrate, although coexisting Alzheimer pathologies are common (about one-third of cases) and independently contribute to dementia in PD.

87. Dauvilliers, Y. et al. REM sleep behaviour disorder. Nat. Rev. Dis. Prim. 4, 19 (2018).

88. Jia, X. et al. Entorhinal cortex atrophy in early, drug-naive Parkinson's disease with mild cognitive impairment. Aging Dis. 10, 1221-1232 (2019).

89. Devanand, D. P. et al. Hippocampal and entorhinal atrophy in mild cognitive impairment: prediction of Alzheimer disease. Neurology 68, 828-836 (2007).

90. Harding, A. J. \& Halliday, G. M. Cortical Lewy body pathology in the diagnosis of dementia. Acta Neuropathol. 102, 355-363 (2001).

91. Dickson, D. W. et al. Neuropathological assessment of Parkinson's disease: refining the diagnostic criteria. Lancet Neurol. 8, 1150-1157 (2009).

92. Yu, L. et al. Common age-related neuropathologies and yearly variability in cognition. Ann. Clin. Transl. Neurol. 6, 2140-2149 (2019).

93. Schaser, A. J. et al. Alpha-synuclein is a DNA binding protein that modulates DNA repair with implications for Lewy body disorders. Sci. Rep. 9, 10919 (2019).

94. Power, J. H., Barnes, O. L. \& Chegini, F. Lewy bodies and the mechanisms of neuronal cell death in Parkinson's disease and dementia with lewy bodies. Brain Pathol. 27, 3-12 (2017).

95. Tagliafierro, L. et al. Genetic analysis of alpha-synuclein 3' untranslated region and its corresponding microRNAs in relation to Parkinson's disease compared to dementia with Lewy bodies. Alzheimers Dement. 13, 1237-1250 (2017)

96. Guella, I. et al. alpha-synuclein genetic variability: a biomarker for dementia in Parkinson disease. Ann. Neurol. 79, 991-999 (2016).

97. Gamez-Valero, A. et al. INDEL length and haplotypes in the beta-synuclein gene: a key to differentiate dementia with Lewy bodies? J. Alzheimers Dis. 65, 207-219 (2018)

98. Tseng, E. et al. The landscape of SNCA transcripts across synucleinopathies: new insights from long reads sequencing analysis. Front. Genet. 10, 584 (2019).

99. Van der Perren, A. et al. The structural differences between patient-derived alpha-synuclein strains dictate characteristics of Parkinson's disease, multiple system atrophy and dementia with Lewy bodies. Acta Neuropathol. 139, 977-1000 (2020). Using homogenized brain proteins from patients dichotomised for different clinical forms of a-synucleinopathies, the authors made pure amplified $\alpha$-synuclein and showed significant differences in the 'strains' of $\alpha$-synuclein in each of the major $\alpha$-synucleinopathies. Signature 'strains' of $\alpha$-synuclein may be diagnostic and also better targeted therapeutically.

100. Candelise, N. et al. Seeding variability of different alpha synuclein strains in synucleinopathies. Ann. Neurol. 85, 691-703 (2019).

101. Petrou, M. et al. Amyloid deposition in Parkinson's disease and cognitive impairment: a systematic review. Mov. Disord. 30, 928-935 (2015).

102. Melzer, T. R. et al. Beta amyloid deposition is not associated with cognitive impairment in Parkinson's disease. Front. Neurol. 10, 391 (2019).

103. Winer, J. R. et al. Associations between Tau, beta-amyloid, and cognition in Parkinson disease. JAMA Neurol. 75, 227-235 (2018). Assessment of $A D$ protein deposition associated with cognitive deficits in PD showing that PD-MCI is not related to the measurable deposition of these proteins, indicating that such protein deposition occurs with progression to dementia in a proportion of patients with PD.

104. Reimand, J. et al. Association of amyloid-beta CSF/PET discordance and tau load 5 years later. Neurology 95, e2648-e2657 (2020).

105. Toledo, J. B. et al. Pathological alpha-synuclein distribution in subjects with coincident Alzheimer's and Lewy body pathology. Acta Neuropathol. 131, 393-409 (2016)

106. Irwin, D. J. et al. Neuropathologic substrates of Parkinson disease dementia. Ann. Neurol. 72, 587-598 (2012)

107. Howard, E. et al. Cognitive profile and markers of AD-type pathology in patients with Lewy body dementias. Neurology 96, e1855-e1864 (2021).

108. Tan, M. M. X. et al. Genome-wide association studies of cognitive and motor progression in Parkinson's disease. Mov. Disord. 36, 424-433 (2021).

109. Iwaki, H. et al. Genomewide association study of Parkinson's disease clinical biomarkers in 12 longitudinal patients' cohorts. Mov. Disord. 34, 1839-1850 (2019).

110. D'Souza, T. \& Rajkumar, A. P. Systematic review of genetic variants associated with cognitive impairment and depressive symptoms in Parkinson's disease. Acta Neuropsychiatr. 32, 10-22 (2020).

111. Hopfner, F. et al. Rare variants in specific lysosomal genes are associated with Parkinson's disease. Mov. Disord. 35, 1245-1248 (2020)

112. Chia, R. et al. Genome sequencing analysis identifies new loci associated with Lewy body dementia and provides insights into its genetic architecture. Nat. Genet. 53, 294-303 (2021).

113. Henderson, M. X. et al. Glucocerebrosidase activity modulates neuronal susceptibility to pathological a-synuclein insult. Neuron 105, 822-836.e7 (2020).

114. Jiang, Z. et al. Characterization of a pathogenic variant in GBA for Parkinson's disease with mild cognitive impairment patients. Mol. Brain 13, 102 (2020).

115. Roussotte, F. F. et al. Carriers of a common variant in the dopamine transporter gene have greater dementia risk, cognitive decline, and faster ventricular expansion. Alzheimers Dement. 11, 1153-1162 (2015).

116. Martelle, S. E. et al. Dopamine pathway gene variants may modulate cognitive performance in the DHS - Mind Study. Brain Behav. 6, e00446 (2016).

117. Caspell-Garcia, C. et al. Multiple modality biomarker prediction of cognitive impairment in prospectively followed de novo Parkinson disease. PLoS One 12, e0175674 (2017).

118. Miranda, G. G Rodrigue K. M \& Kennedy, K. M. Frontoparietal cortical thickness mediates the effect of COMT Val(158)Met polymorphism on age-associated executive function. Neurobiol. Aging 73, 104-114 (2019).

119. Backstrom, D. et al. Polymorphisms in dopamine associated genes and cognitive decline in Parkinson's disease. Acta Neurol. Scand. 137, 91-98 (2018).

120. Skorvanek, M. et al. Global scales for cognitive screening in Parkinson's disease: critique and recommendations. Mov. Disord. 33, 208-218 (2018).

121. Nasreddine, Z. S. et al. The Montreal Cognitive Assessment, MoCA: a brief screening tool for mild cognitive impairment. J. Am. Geriatr. Soc. 53 , 695-699 (2005).

122. Mattis, S. Dementia Rating Scale: Professional Manual (Psychological Assessment Resources Incorporated, 1988).

123. Pagonabarraga, J. et al. Parkinson's disease-cognitive rating scale: a new cognitive scale specific for Parkinson's disease. Mov. Disord. 23, 998-1005 (2008).

124. Caslake, R. et al. The mini-mental Parkinson's (MMP) as a cognitive screening tool in people with Parkinson's disease. Curr. Aging Sci. 6, 273-279 (2013)

125. Marinus, J. et al. Assessment of cognition in Parkinson's disease. Neurology 61, 1222 (2003).

126. Hoops, S. et al. Validity of the MoCA and MMSE in the detection of $\mathrm{MCl}$ and dementia in Parkinson disease. Neurology 73, 1738-1745 (2009).

127. Marras, C. et al. Measuring mild cognitive impairment in patients with Parkinson's disease. Mov. Disord. 28, 626-633 (2013)

128. Hoogland, J. et al. Detecting mild cognitive deficits in Parkinson's disease: comparison of neuropsychological tests. Mov. Disord. 33, 1750-1759 (2018).

129. Chaudhuri, K. R. et al. The metric properties of a novel non-motor symptoms scale for Parkinson's disease: results from an international pilot study. Mov. Disord. 22, 1901-1911 (2007) 
130. Chaudhuri, K. R et al. The Movement Disorder Society Nonmotor Rating Scale: initial validation study. Mov. Disord. 35, 116-133 (2020).

131. Chaudhuri, K. R. et al. International multicenter pilot study of the first comprehensive self-completed nonmotor symptoms questionnaire for Parkinson's disease: the NMSQuest study. Mov. Disord. 21 916-923 (2006)

132. Kulisevsky, J. et al. Measuring functional impact of cognitive impairment: validation of the Parkinson's disease cognitive functional rating scale. Parkinsonism Relat. Disord. 19, 812-817 (2013).

133. Brennan, L. et al. The Penn Parkinson's Daily Activities Questionnaire-15: psychometric properties of a brief assessment of cognitive instrumental activities of daily living in Parkinson's disease. Parkinsonism Relat. Disord. 25, 21-26 (2016).

134. Hoogland, J. et al. Risk of Parkinson's disease dementia related to level I MDS PD-MCI. Mov. Disord. 34, 430-435 (2019)

Validation of the level I and level II PD-MCI criteria by showing their predictive value for PDD in a large combination of cohorts.

135. Hoogland, J. et al. Mild cognitive impairment as a risk factor for Parkinson's disease dementia. Mov. Disord. 32, 1056-1065 (2017)

136. Saredakis, D. Collins-Praino, L. E., Gutteridge, D. S. Stephan, B. C. M. \& Keage, H. A. D. Conversion to $\mathrm{MCl}$ and dementia in Parkinson's disease: a systematic review and meta-analysis. Parkinsonism Relat. Disord. 65, 20-31 (2019).

137. Baiano, C., Barone, P., Trojano, L. \& Santangelo, G. Prevalence and clinical aspects of mild cognitive impairment in Parkinson's disease: a meta-analysis. Mov. Disord. 35, 45-54 (2020).

Meta-analysis of the prevalence rate of PD-MC according to the MDS clinical criteria. Furthermore, an exploration of the differences between patients with and without PD-MCl in demographic, clinical and neuropsychiatric features.

138. Rosen, W. G., Mohs, R. C. \& Davis, K. L. A new rating scale for Alzheimer's disease. Am. J. Psychiatry 141 1356-1364 (1984).

139. Galasko, D. et al. An inventory to assess activities of daily living for clinical trials in Alzheimer's disease. The Alzheimer's Disease Cooperative Study. Alzheimer Dis. Assoc. Disord. 11 (Suppl. 2), S33-S39 (1997).

140. Schneider, L. S. et al. Validity and reliability of the Alzheimer's disease cooperative study-clinical global impression of change. The Alzheimer's Disease Cooperative Study. Alzheimer Dis. Assoc. Disord. 11 (Suppl. 2), S22-S32 (1997)

141. Arvanitakis, Z., Shah, R. C. \& Bennett, D. A. Diagnosis and management of dementia: review. JAMA 322 1589-1599 (2019)

142. Burton, E. J., McKeith, I. G., Burn, D. J., Williams, E. D. \& O’Brien, J. T. Cerebral atrophy in Parkinson's disease with and without dementia: a comparison with Alzheimer's disease, dementia with Lewy bodies and controls. Brain 127, 791-800 (2004)

143. Hwang, K. S. et al. Mapping cortical atrophy in Parkinson's disease patients with dementia. J. Parkinsons Dis. 3, 69-76 (2013)

144. Dubois, B. et al. Diagnostic procedures for Parkinson's disease dementia: recommendations from the movement disorder society task force. Mov. Disord. 22, 2314-2324 (2007)

145. Smirnov, D S et al Cognitive decline profiles differ in Parkinson disease dementia and dementia with Lewy bodies. Neurology 94, e2076-e2087 (2020).

146. Petrova, M., Mehrabian-Spasova, S., Aarsland, D. Raycheva, M. \& Traykov, L. Clinical and neuropsychological differences between mild Parkinson's disease dementia and dementia with Lewy bodies. Dement. Geriatr. Cogn. Disord. Extra 5, 212-220 (2015)

147. Owens, A. P. et al. Implementing remote memory clinics to enhance clinical care during and after COVID-19. Front. Psychiatry 11, 579934 (2020).

148. Brooker, H. et al. FLAME: a computerized neuropsychological composite for trials in early dementia. Alzheimers Dement 12, e12098 (2020).

149. Owens, A. P. et al. Selecting remote measurement technologies to optimize assessment of function in early Alzheimer's disease: a case study. Front. Psychiatry 11, 1163 (2020).

150. van den Berg, E., Ruis, C., Biessels, G. J., Kappelle, L. J. $\&$ van Zandvoort, M. J. The Telephone Interview for Cognitive Status (Modified): relation with a comprehensive neuropsychological assessment. J. Clin. Exp. Neuropsychol. 34, 598-605 (2012).
151. Marra, D. E., Hamlet, K. M., Bauer, R. M. \& Bowers, D. Validity of teleneuropsychology for older adults in response to COVID-19: A systematic and critical review. Clin. Neuropsychol. 34, 1411-1452 (2020).

152. Phillips, N. A Chertkow, H., Pichora-Fuller, M. K. ¿ Wittich, W. Special issues on using the Montreal Cognitive Assessment for telemedicine Assessment during COVID-19. J. Am. Geriatrics Soc. 68, 942-944 (2020).

153. Schrag, A., Siddiqui, U. F., Anastasiou, Z., Weintraub, D. $\&$ Schott, J. M. Clinical variables and biomarkers in prediction of cognitive impairment in patients with newly diagnosed Parkinson's disease: a cohort study. Lancet Neurol. 16, 66-75 (2017).

154. Weintraub, D. et al. Alzheimer's disease pattern of brain atrophy predicts cognitive decline in Parkinson's disease. Brain 135, 170-180 (2012).

155. Huang, C. et al. Metabolic brain networks associated with cognitive function in Parkinson's disease. Neuroimage 34, 714-723 (2007).

156. Lanskey, J. H. et al. Can neuroimaging predict dementia in Parkinson's disease? Brain 141 2545-2560 (2018).

157. Zhang, Y., Wu, I. W., Tosun, D., Foster, E. \& Schuff, N. Progression of regional microstructural degeneration in Parkinson's disease: a multicenter diffusion tensor imaging study. PLoS One 11, e0165540 (2016).

158. Siderowf, A. et al. CSF amyloid \{beta\} 1-42 predicts cognitive decline in Parkinson disease. Neurology 75 1055-1061 (2010).

159. Johar, I., Mollenhauer, B. \& Aarsland, D. Cerebrospinal fluid biomarkers of cognitive decline in Parkinson's disease. Int. Rev. Neurobiol. 132, 275-294 (2017).

160. Yousaf, T., Pagano, G., Niccolini, F. \& Politis, M. Predicting cognitive decline with non-clinical markers in Parkinson's disease (PRECODE-2). J. Neurol. 266, 1203-1210 (2019)

161. Hansen, A. K. et al. Tau tangles in Parkinson's disease: a 2-year follow-up flortaucipir PET study. $J$ Parkinsons Dis. 10, 161-171 (2020)

162. Lin, C. H. \& Wu, R. M. Biomarkers of cognitive decline in Parkinson's disease. Parkinsonism Relat. Disord. 21, 431-443 (2015)

163. Mavroudis, I., Petridis, F., Chatzikonstantinou, S. \& Kazis, D. Alpha-synuclein levels in the differential diagnosis of Lewy bodies dementia and other neurodegenerative disorders: a meta-analysis. Alzheimer Dis. Assoc. Disord. 34, 220-224 (2020).

164. Paciotti, S., Bellomo, G., Gatticchi, L. \& Parnetti, L. Are we ready for detecting $\alpha$-synuclein prone to aggregation in patients? The case of "protein-misfolding cyclic amplification" and "real-time quaking-induced conversion" as diagnostic tools. Front. Neurol. 9 415 (2018).

165. Massa, F. et al. Utility of quantitative EEG in early Lewy body disease. Parkinsonism Relat. Disord. 75 70-75 (2020).

166. Betrouni, N. et al. Electroencephalography-based machine learning for cognitive profiling in Parkinson's disease: preliminary results. Mov. Disord. 34 210-217 (2019).

167. Bonanni, L. et al. EEG comparisons in early Alzheimer's disease, dementia with Lewy bodies and Parkinson's disease with dementia patients with a 2-year follow-up. Brain 131, 690-705 (2008).

168. Klassen, B. T. et al. Quantitative EEG as a predictive biomarker for Parkinson disease dementia. Neurology 77, 118-124 (2011)

169. Emre, M. et al. Rivastigmine for dementia associated with Parkinson's disease. N. Engl. J. Med. 351 , 2509-2518 (2004).

170. Mamikonyan, E., Xie, S. X., Melvin, E. \& Weintraub, D. Rivastigmine for mild cognitive impairment in Parkinson disease: a placebo-controlled study. Mov. Disord. 30, 912-918 (2015).

171. Emre, M. et al. Long-term safety of rivastigmine in Parkinson disease dementia: an open-label, randomized study. Clin. Neuropharmacol. 37, 9-16 (2014).

172. Dubois, B. et al. Donepezil in Parkinson's disease dementia: a randomized double-blind efficacy and safety study. Mov. Disord. 27, 1230-1238 (2012).

173. Aarsland, D. et al. Memantine in patients with Parkinson's disease dementia or dementia with Lewy bodies: a double-blind, placebo-controlled, multicentre trial. Lancet Neurol. 8, 613-618 (2009).

174. Emre, M. et al. Memantine for patients with Parkinson's disease dementia or dementia with Lewy bodies: a randomized, double-blind, placebo-controlled trial Lancet Neurol 9, 969-977(2010).
175. Wang, H. F. et al. Efficacy and safety of cholinesterase inhibitors and memantine in cognitive impairment in Parkinson's disease, Parkinson's disease dementia, and dementia with Lewy bodies: systematic review with meta-analysis and trial sequential analysis. J. Neurol. Neurosurg. Psychiatry 86, 135-143 (2015).

176. Taylor, J. P. et al. New evidence on the management of Lewy body dementia. Lancet Neurol. 19, 157-169 (2020).

Review addressing existing gaps in the management of Lewy body dementia (DLB and PDD) and covering the current management of key domains of cognitive impairment, neuropsychiatric symptom as well as motor and other non-motor symptoms.

177. Litvan, I., Kieburtz, K., Tröster, A. I. \& Aarsland, D. Strengths and challenges in conducting clinical trials in Parkinson's disease mild cognitive impairment. Mov. Disord. 33, 520-527 (2018).

178. Weintraub, D. et al. Rasagiline for mild cognitive impairment in Parkinson's disease: a placebo-controlled trial. Mov. Disord. 31, 709-714 (2016).

179. Sawada, H. et al. Early use of donepezil against psychosis and cognitive decline in Parkinson's disease: a randomised controlled trial for 2 years. J. Neurol. Neurosurg. Psychiatry 89, 1332-1340 (2018).

180. Weintraub, D. et al. Atomoxetine for depression and other neuropsychiatric symptoms in Parkinson's disease. Neurology 75, 448-455 (2010).

181. Kehagia, A. et al. Targeting impulsivity in Parkinson's disease using atomoxetine. Brain 137, 1986-1997 (2014).

182. Hinson, V. K., Delambo, A., Elm, J. \& Turner, T. A randomized clinical trial of atomoxetine for mild cognitive impairment in Parkinson's disease. Mov. Disord. Clin. Pract. 4, 416-423 (2017)

183. Biundo, R., Weis, L., Fiorenzato, E. \& Antonini, A. Cognitive rehabilitation in Parkinson's disease: is it feasible? Arch. Clin. Neuropsychol. 32, 840-860 (2017).

184. Lawrence, B. J., Gasson, N., Bucks, R. S., Troeung, L. $\&$ Loftus, A. M. Cognitive training and noninvasive brain stimulation for cognition in Parkinson's disease: a meta-analysis. Neurorehabil Neural Repair. 31, 597-608 (2017).

185. Leung, I. H. et al. Cognitive training in Parkinson disease: a systematic review and meta-analysis. Neurology 85, 1843-1851 (2015).

186. Orgeta, V. et al. Cognitive training interventions for dementia and mild cognitive impairment in Parkinson's disease. Cochrane Database Syst. Rev. 2, CD011961 (2020).

Systematic review investigating whether cognitive training improves cognition in people with PDD and PD-MCl or other clearly defined forms of cognitive impairment in people with PD.

187. Lauze, M. Daneault J. F \& Duval C. The effects of physical activity in Parkinson's disease: a review. J. Parkinsons Dis. 6, 685-698 (2016).

188. da Silva, F. C. et al. Effects of physical exercise programs on cognitive function in Parkinson's disease patients: a systematic review of randomized controlled trials of the last 10 years. PLoS One 13, e0193113 (2018) Systematic review aiming to determine the effects of physical exercise programmes on cognitive function in patients with PD.

189. Dinkelbach, L. Brambilla M. Manenti, R \& Brem, A. K. Non-invasive brain stimulation in Parkinson's disease: exploiting crossroads of cognition and mood. Neurosci. Biobehav. Rev. 75, 407-418 (2017).

190. Nousia, A et al. The beneficial effects of computer-based cognitive training in Parkinson's disease: a systematic review. Arch. Clin. Neuropsychol. 35, 434-447 (2020)

191. Stuckenschneider, T. et al. The effect of different exercise modes on domain-specific cognitive function in patients suffering from Parkinson's disease: a systematic review of randomized controlled trials. J. Parkinsons Dis. 9, 73-95 (2019).

192. Lefaucheur, J. P. et al. Evidence-based guidelines on the therapeutic use of transcranial direct current stimulation (tDCS). Clin. Neurophysiol. 128, 56-92 (2017).

193. Auvichayapat, P. \& Auvichayapat, N. Basic principle of transcranial magnetic stimulation. J. Med. Assoc. Tha 92, 1560-1566 (2009).

194. Gratwicke, J. et al. Bilateral deep brain stimulation of the nucleus basalis of Meynert for Parkinson disease dementia: a randomized clinical trial. JAMA Neurol. 75, 169-178 (2018) 
195. PD Med Collaborative Group, et al.Long-term effectiveness of dopamine agonists and monoamine oxidase B inhibitors compared with levodopa as initial treatment for Parkinson's disease (PD MED): a large, open-label, pragmatic randomised trial. Lancet 384 1196-1205 (2014)

196. Verschuur, C. V. M. et al. Randomized delayed-start trial of levodopa in Parkinson's disease. N. Engl. J. Med. 380, 315-324 (2019).

197. Abraham, D. S. et al. Frequency of and risk factors for potentially inappropriate medication use in Parkinson's disease. Age Ageing 49, 786-792 (2020).

198. Mantri, S. et al. Patterns of dementia treatment and frank prescribing errors in older adults with Parkinson disease. JAMA Neurol. 76, 41-49 (2019).

199. Ehrt, U., Broich, K., Larsen, J., Ballard, C \& Aarsland, D. Use of drugs with anticholinergic effect and impact on cognition in Parkinson's disease: a cohort study. J. Neurol. Neurosurg. Psychiatry $\mathbf{8 1}$ 160-165 (2010).

200. Witt, K et al. Relation of lead trajectory and electrode position to neuropsychological outcomes of subthalamic neurostimulation in Parkinson's disease: results from a randomized trial. Brain 136 2109-2119 (2013)

201. Frankemolle, A. et al. Reversing cognitive-motor impairments in Parkinson's disease patients using a computational modelling approach to deep brain stimulation programming. Brain 133, 746-761 (2010).

202. Schuepbach, W. M. et al. Neurostimulation for Parkinson's disease with early motor complications. N. Engl. J. Med. 368, 610-622 (2013).

203. Trenkwalder, C. et al. Expert Consensus Group report on the use of apomorphine in the treatment of Parkinson's disease - clinical practice recommendations. Parkinsonism Relat. Disord. 21, 1023-1030 (2015)

204. Timpka, J., Nitu, B., Datieva, V., Odin, P. \& Antonini, A. Device-aided treatment strategies in advanced Parkinson's disease. Int. Rev. Neurobiol. 132, 453-474 (2017)

205. Chahine, L. M. et al. The most bothersome aspects of off periods reported by individuals with Parkinson's disease. Mov. Disord. Clin. Pract. 7, 284-292 (2020).

206. Witjas, T. et al. Nonmotor fluctuations in Parkinson's disease: frequent and disabling. Neurology 59 , 408-413 (2002)

207. Chahine, L. M. et al. Modifiable vascular risk factors, white matter disease and cognition in early Parkinson's disease. Eur. J. Neurol. 26, 246-e18 (2019).

208. Mollenhauer, B. et al. Baseline predictors for progression 4 years after Parkinson's disease diagnosis in the De Novo Parkinson Cohort (DeNoPa). Mov. Disord. 34, 67-77 (2019).

209. Knox, M. G. et al. Neuropathological findings in Parkinson's disease with mild cognitive impairment. Mov. Disord. 35, 845-850 (2020).

210. Centi, J. et al. Effects of orthostatic hypotension on cognition in Parkinson disease. Neurology 88, 17-24 (2017).

211. Harmell, A. L. et al. Obstructive sleep apnea and cognition in Parkinson's disease. Sleep. Med. 21, 28-34 (2016).

212. Kaminska, M. et al. Change in cognition and other non-motor symptoms with obstructive sleep apnea treatment in Parkinson disease. J. Clin. Sleep. Med. 14, 819-828 (2018).

213. Jester, D. J., Lee, S., Molinari, V. \& Volicer, L. Cognitive deficits in Parkinson's disease with excessive daytime sleepiness: a systematic review. Aging Ment. Health 24, 1769-1780 (2020)

214. Li, Z. et al. The effect of creatine and coenzyme q10 combination therapy on mild cognitive impairmen in Parkinson's disease. Eur. Neurol. 73, 205-211 (2015)

215. Varela-López, A., Giampieri, F., Battino, M. \& Quiles, J. L. Coenzyme $\mathrm{Q}$ and its role in the dietary therapy against aging. Molecules 21, 373 (2016).

216. Andres, R. H., Ducray, A. D., Schlattner, U., Wallimann, T. \& Widmer, H. R. Functions and effects of creatine in the central nervous system. Brain Res. Bull. 76, 329-343 (2008)

217. Mo, J. J. et al. The effectiveness of creatine treatment for Parkinson's disease: an updated meta-analysis of randomized controlled trials. BMC Neurol. 17, 105 (2017).

218. Parkinson Study Group QE3 Investigators et al. A randomized clinical trial of high-dosage coenzyme
Q10 in early Parkinson disease: no evidence of benefit. JAMA Neurol. 71, 543-552 (2014).

219. Svenningsson, P. et al. A phase 2 a trial investigating the safety and tolerability of the novel cortical enhancer IRL752 in Parkinson's disease dementia. Mov. Disord. 35, 1046-1054 (2020).

Randomized placebo-controlled trial showing safety and tolerability data for a novel compound producing regioselective enhancement of cortical noradrenaline, dopamine and acetylcholine.

220. Aarsland, D. et al. ANAVEX ${ }^{\circledR 2-73}$ (blarcamesine) Currently in Phase 2b/3 Early Alzheimer's Disease $(A D)$ : analysis of cognitive outcome measures relevant in $\mathrm{AD}$ of double-blind, multicenter, placebo-controlled phase 2 clinical trial in 132 patients with Parkinson's disease dementia. https://www.anavex.com/ wp-content/uploads/2020/11/CTAD-Anavex-PDD-

001-Topline-data-Presentation-2020.pdf (2020).

221. Alam, J. J. et al. The p38a kinase inhibito neflamapimod significantly improves cognition in patients with mild-to-moderate dementia with Lewy bodies (DLB) [abstract LB22]. J. Prev. Alzheimers Dis. 7, S2-S54, (2020).

222. Biundo, R. et al. Double-blind randomized trial of tDCS versus sham in Parkinson patients with mild cognitive impairment receiving cognitive training. Brain Stimul. 8, 1223-1225 (2015).

223. Manenti, R. et al. Mild cognitive impairment in Parkinson's disease is improved by transcranial direct current stimulation combined with physical therapy. Mov. Disord. 31, 715-724 (2016).

224. Willis, A. W. et al. Predictors of survival in patients with Parkinson disease. Arch. Neurol. 69, 601-607 (2012).

225. Backstrom, D. et al. Early predictors of mortality in parkinsonism and Parkinson disease: a population-based study. Neurology 91, e2045-e2056 (2018).

226. Martinez-Martin, P., Rodriguez-Blazquez, C., Kurtis, M. M., Chaudhuri, K. R. \& Group, N. V. The impact of non-motor symptoms on health-related quality of life of patients with Parkinson's disease. Mov. Disord. 26, 399-406 (2011). Study using the NMSS to assess the impact of non-motor symptoms on the HROOL of patients with PD.

227. Martinez-Martin, P. An introduction to the concept of "quality of life in Parkinson's disease". J. Neurol. 245 S2-S6 (1998)

228. Peto, V., Jenkinson, C., Fitzpatrick, R. \& Greenhall, R. The development and validation of a short measure of functioning and well being for individuals with Parkinson's disease. Qual. Life Res. 4, 241-248 (1995)

229. Jenkinson, C., Fitzpatrick, R., Peto, V., Greenhall, R. $\&$ Hyman, N. The PDQ-8: development and validation of a short-form parkinson's disease questionnaire. Psychol. Health 12, 805-814 (1997).

230. EuroOol Group. EuroQol - a new facility for the measurement of health-related quality of life. Health Policy 16, 199-208 (1990)

231. Zarit, S. H., Reever, K. E. \& Bach-Peterson, J. Relatives of the impaired elderly: correlates of feelings of burden. Gerontologist 20, 649-655 (1980)

232. Yu, J., Yap, P. \& Liew, T. M. The optimal short version of the Zarit Burden Interview for dementia caregivers: diagnostic utility and externally validated cutoffs. Aging Ment. Health 23, 706-710 (2019).

233. Hurt, C. S. et al. Identifying barriers to help-seeking for non-motor symptoms in people with Parkinson's disease. J. Health Psychol. 24, 561-571 (2019).

234. Lawson, R. A. et al. Cognitive decline and quality of life in incident Parkinson's disease: the role of attention Parkinsonism Relat. Disord. 27 47-53 (2016).

235. Morley, D. et al. Factors influencing quality of life in caregivers of people with Parkinson's disease and implications for clinical guidelines. Parkinsons Dis. 2012, 190901 (2012).

236. Forsaa, E. B et al. A 12-year population-based study of psychosis in Parkinson disease. Arch. Neurol. 67, 996-1001 (2010).

237. Pedersen, K. F., Larsen, J. P., Alves, G. \& Aarsland, D. Prevalence and clinical correlates of apathy in Parkinson's disease: a community-based study. Parkinsonism Relat. Disord. 15, 295-299 (2009).

238. Riedel, O. et al. Frequency of dementia, depression, and other neuropsychiatric symptoms in 1,449 outpatients with Parkinson's disease. J. Neurol. 257, 1073-1082 (2010)

239. Martinez-Martin, P., Wan, Y. M., Ray Chaudhuri, K., Schrag, A. E. \& Weintraub, D. Impulse control and related behaviors in Parkinson's disease with dementia. Eur. J. Neurol. 27, 944-950 (2020).
240. Martinez-Martin, P. et al. Neuropsychiatric symptoms and caregiver's burden in Parkinson's disease. Parkinsonism Relat. Disord. 21, 629-634 (2015).

241. Karlstedt, M., Fereshtehnejad, S. M., Aarsland, D. \& Lökk, J. Mediating effect of mutuality on caregiver burden in Parkinson's disease partners. Aging Ment Health 24, 1421-1428 (2020).

242. Vatter, S., McDonald, K. R., Stanmore, E., Clare, L. $\&$ Leroi, I. Multidimensional care burden in Parkinson-related dementia. J. Geriatr. Psychiatry Neurol. 31, 319-328 (2018).

243. Vatter, S. et al. Care burden and mental III health in spouses of people with Parkinson disease dementia and Lewy body dementia. J. Geriatr. Psychiatry Neurol. 33, 3-14 (2020).

244. Leta, V. et al. Parkinson's disease and post-COVID-19 syndrome: the Parkinson's long-COVID spectrum. Mov. Disord. https://doi.org/10.1002/mds.28622 (2021).

245. Feigin, V. L. et al. Global, regional, and national burden of neurological disorders during 1990-2015: a systematic analysis for the Global Burden of Disease Study 2015. Lancet Neurol. 16, 877-897 (2017).

246. Dorsey, E. R. et al. Global, regional, and national burden of Parkinson's disease, 1990-2016: a systematic analysis for the Global Burden of Disease Study 2016. Lancet Neurol. 17, 939-953 (2018)

247. Dorsey, E. R. \& Bloem, B. R. The Parkinson pandemic-a call to action. JAMA Neurol. 75, 9-10 (2018).

248. Williams, U., Bandmann, O. \& Walker, R. Parkinson's disease in sub-Saharan Africa: a review of epidemiology, genetics and access to care. J. Mov. Disord. 11, 53-64 (2018).

249. Khalil, H., Chahine, L., Siddiqui, J., Aldaajani, Z. \& Bajwa, J. A. Parkinson's disease in the MENASA countries. Lancet Neurol. 19, 293-294 (2020).

250. Kaiyrzhanov, R. et al. Parkinson's disease in central Asian and transcaucasian countries: a review of epidemiology, genetics, clinical characteristics, and access to care. Parkinsons Dis. 2019, 2905739 (2019)

251. Nichols, E. et al. Global, regional, and national burden of Alzheimer's disease and other dementias, 1990-2016: a systematic analysis for the Global Burden of Disease Study 2016. Lancet Neurol. 18, 88-106 (2019).

252. Giebel, C. "Current dementia care: what are the difficulties and how can we advance care globally?". BMC Health Serv. Res. 20, 414 (2020).

253. Ferri, C. P. \& Jacob, K. S. Dementia in low-income and middle-income countries: different realities mandate tailored solutions. PLoS Med. 14, e1002271 (2017).

254. Dekker, M. C. J. et al. Parkinson's disease research on the African continent: obstacles and opportunities. Front. Neurol. 11, 512 (2020).

255. Stephan, B. C. M. et al. Prediction of dementia risk in low-income and middle-income countries (the 10/66 Study): an independent external validation of existing models. Lancet Glob. Health 8, e524-e535 (2020).

256. Berg, D. et al. Time to redefine PD? Introductory statement of the MDS Task Force on the definition of Parkinson's disease. Mov. Disord. 29, 454-462 (2014).

257. Boeve, B. F. et al. Arguing against the proposed definition changes of PD. Mov. Disord. 31 1619-1622 (2016).

258. Postuma, R. B. et al. Abolishing the 1-year rule: how much evidence will be enough? Mov. Disord. 31 1623-1627 (2016).

259. Friedman, J. H. Dementia with Lewy bodies and Parkinson disease dementia: it is the same disease! Parkinsonism Relat. Disord. 46, S6-S9 (2018).

260. Jellinger, K. A. Dementia with Lewy bodies and Parkinson's disease-dementia: current concepts and controversies. J. Neural Transm. 125, 615-650 (2018).

Critical review on the current concepts and controversies in the pathogenesis of DLB and PDD and their possible nosological interrelations, available biological markers, and present and future treatment strategies.

261. Chahine, L. M. et al. Cognition among individuals along a spectrum of increased risk for Parkinson's disease. PLoS One 13, e0201964 (2018). Study conducted on patients with de novo PD, with idiopathic RBD, with hyposmia, and non-PD LRRK2 and $C B A$ mutation carriers showing that cognitive function is worse among those with RBD, the characteristic most strongly associated with future risk of PD or dementia with Lewy bodies.

262. Rahayel, S. et al. A prodromal brain-clinical pattern of cognition in synucleinopathies. Ann. Neurol. 89, 341-357 (2021) 
263. Chahine, L. M. et al. In vivo distribution of $\alpha$-synuclein in multiple tissues and biofluids in Parkinson disease. Neurology 95, e1267-e1284 (2020).

264. Pilotto, A. et al. Plasma NfL, clinical subtypes and motor progression in Parkinson's disease. Parkinsonism Relat. Disord. 87, 41-47 (2021).

265. Lin, C. H. et al. Plasma alpha-synuclein predicts cognitive decline in Parkinson's disease. J. Neurol. Neurosurg. Psychiatry 88, 818-824 (2017).

266. Schmand, B. Why are neuropsychologists so reluctant to embrace modern assessment techniques? Clin. Neuropsychol. 33, 209-219 (2019).

267. McFarthing, K. et al. Parkinson's disease drug therapies in the clinical trial pipeline: 2020. J. Parkinsons Dis. 10, 757-774 (2020).

268. Mandler, M. et al. Effects of single and combined immunotherapy approach targeting amyloid beta protein and alpha-synuclein in a dementia with Lewy bodies-like model. Alzheimers Dement. 15, 1133-1148 (2019)

269. Valera, E., Spencer, B. \& Masliah, E. Immunotherapeutic approaches targeting amyloid- $\beta$, $\alpha$-synuclein, and Tau for the treatment of neurodegenerative disorders Neurotherapeutics 13, 179-189 (2016).

270. Takamatsu, Y. et al. Combined immunotherapy with "anti-insulin resistance" therapy as a novel therapeutic strategy against neurodegenerative diseases. NPJ Parkinsons Dis. 3, 4 (2017).

271. Kwon, S., Iba, M., Kim, C. \& Masliah, E. Immunotherapies for aging-related neurodegenerative diseases-emerging perspectives and new targets. Neurotherapeutics 17, 935-954 (2020).

272. Green, H., Tsitsi, P., Markaki, I., Aarsland, D. \& Svenningsson, P. Novel treatment opportunities against cognitive impairment in Parkinson's disease with an emphasis on diabetes-related pathways. CNS Drugs 33, 143-160 (2019).

Review of ongoing clinical trials in patients with PD and dementia, highlighting the multiple pharmacological mechanisms that are targeted to achieve cognitive enhancement, with a specific focus on diabetes-related pathways.

273. Hung, A. Y. \& Schwarzschild, M. A. Approaches to disease modification for Parkinson's disease: clinica trials and lessons learned. Neurotherapeutics 17 1393-1405 (2020)

274. Hughes, M. \& Duffy, C. Public involvement in health and social sciences research: a concept analysis. Health Expect. 21, 1183-1190 (2018).

275. Qamar, M. A., Rizos, A., Stones, L., Meachin, C. \& Chaudhuri, K. R. Public and patient involvement (PPI) at King's: community for research involvement and support for people with Parkinson's (CRISP). ACNR 16, 17-20 (2016).

276. de Roos, P. et al. A consensus set of outcomes for Parkinson's disease from the international consortium for health outcomes measurement. J. Parkinsons Dis. 7, 533-543 (2017).

277. Aarsland, D. et al. Cognitive impairment in incident, untreated Parkinson disease: the Norwegian ParkWest study. Neurology 72, 1121-1126 (2009).
278. Broeders, M. et al. Evolution of mild cognitive impairment in Parkinson disease. Neurology 81 , 346-352 (2013).

279. Pigott, K. et al. Longitudinal study of normal cognition in Parkinson disease. Neurology 85, 1276-1282 (2015).

280. Weintraub, D. et al. Neuropsychiatric symptoms and cognitive abilities over the initial quinquennium of Parkinson disease. Ann. Clin. Transl. Neurol. 7, 449-461 (2020)

281. Leroi, I. et al. Randomized placebo-controlled trial of donepezil in cognitive impairment in Parkinson's disease. Int. J. Geriatr. Psychiatry 19, 1-8 (2004).

282. Ravina, B. et al. Donepezil for dementia in Parkinson's disease: a randomised, double blind, placebo controlled, crossover study. J. Neurol. Neurosurg. Psychiatry 76, 934-939 (2005).

283. Litvinenko, I. V., Odinak, M. M., Mogil'naya, V. I. $\ltimes$ Emelin, A. Y. Efficacy and safety of galantamine (reminyl) for dementia in patients with Parkinson's disease (an open controlled trial). Neurosci. Behav. Physiol. 38, 937-945 (2008).

284. Leroi, I., Overshott, R., Byrne, E. J., Daniel, E. \& Burns, A. Randomized controlled trial of memantine in dementia associated with Parkinson's disease. Mov. Disord. 24, 1217-1221 (2009).

285. Cerasa A \& Quattrone, A. The effectiveness of cognitive treatment in patients with Parkinson's disease: a new phase for the neuropsychological rehabilitation. Parkinsonism Relat. Disord. 21, 165 (2015).

286. Alloni, A. Quaglini, S., Panzarasa, S., Sinforiani, E. \& Bernini, S. Evaluation of an ontology-based system for computerized cognitive rehabilitation. Int. J. Med. Inf. 115, 64-72 (2018).

287. Paris, A. P. et al. Blind randomized controlled study of the efficacy of cognitive training in Parkinson's disease Mov. Disord. 26, 1251-1258 (2011).

288. Lawrence, B. J., Gasson, N., Johnson, A. R. Booth, L. \& Loftus, A. M. Cognitive training and transcranial direct current stimulation for mild cognitive impairment in Parkinson's disease: a randomized controlled trial. Parkinsons Dis. 2018. 4318475 (2018).

289. Hanagasi, H. et al. The effects of rasagiline on cognitive deficits in Parkinson's disease patients without dementia: a randomized, double-blind placebo-controlled, multicenter study. Mov. Disord. 26, 1851-1858 (2011)

290. Jack, C. R. Jr. et al. NIA-AA research framework: toward a biological definition of Alzheimer's disease. Alzheimers Dement. 14, 535-562 (2018).

291. Ma K -L et al. The nuclear accumulation of alpha-synuclein is mediated by importin alpha and promotes neurotoxicity by accelerating the cell cycle. Neuropharmacology 82, 132-142 (2014).

\section{Acknowledgements}

D.A. is a Royal Society Wolfson Research Merit Award Holde and would like to thank the Wolfson Foundation and the Royal Society for their support.

\section{Author contributions}

Introduction (D.A. L.B. and D.W.) Epidemiology (D.A., L.B., C.B., G.M.H. and D.W.); Mechanisms/pathophysiology (G.M.H. D.A. and D.W.); Diagnosis, screening and prevention (G.J.G. and D.A.); Management (D.W., L.B. and D.A.); Quality of life (K R. C. D.A and L B): Outlook (All): Overview of Primer (D.A. and L.B.). D.A. and L.B. contributed equally to this work.

\section{Competing interests}

This paper represents independent research partly funded by the National Institute for Health Research (NIHR) Biomedical Research Centre at South London and Maudsley NHS Foundation Trust and King's College London. The views expressed are those of the author and not necessarily those of the NHS, the NIHR or the Department of Health and Social Care. D.A. has received research support and/or honoraria from Astra-Zeneca, H. Lundbeck, Novartis Pharmaceuticals, Evonik, and GE Health and has served as paid consultant for H. Lundbeck, Eisai, Heptares, Mentis Cura, Eli Lilly, and Biogen. C.B. reports grants and personal fees from Acadia pharmaceutical company, grants and personal fees from Lundbeck, personal fees from Roche, personal fees from Otsuka, personal fees from Biogen, personal fees from Eli Lilly, personal fees from Novo Nordisk, personal fees from AARP, grants and personal fees from Synexus, and personal fees from Exciva outside the submitted work. K.R.C. is in the advisory board of AbbVie, UCB, GKC, Bial, Cynapsus, Lobsor, Stada, Medtronic, Zambon, Profile, Sunovion, Roche, Therevance, Scion, Britannia, Acadia and 4D, has received honoraria for lectures from AbbVie, Britannia, UCB, Zambon, Novartis, Boeringer Ingelheim and Bial, and reports grants for investigator-initiated studies from Britania Pharmaceuticals, AbbVie, UCB, GKC and Bial as well as academic grants from EU, IMI EU, Horizon 2020, Parkinson's UK, NIHR, PDNMG, Kirby Laing Foundation, NPF, MRC, and Wellcome Trust. D.W. has received research funding or support from Michael J. Fox Foundation for Parkinson's Research, Alzheimer's Therapeutic Research Initiative (ATRI), Alzheimer's Disease Cooperative Study (ADCS), the International Parkinson and Movement Disorder Society (IPMDS) and the National Institute on Ageing (NIA), honoraria for consultancy from Acadia, Aptinyx, CHDI Foundation, Clintrex LLC (Alkahest, Aptinyx, Avanir, Otsuka), Eisai, Enterin, Great Lake Neurotechnologies, Janssen, Sage, Scion, Signant Health, Sunovion and Vanda, and license fee payments from the University of Pennsylvania for the QUIP and QUIP-RS. L.B., G.H. and G.G. report no competing interests.

Peer review information

Nature Reviews Disease Primers thanks P. H. Lee (who co-reviewed this manuscript with $\mathrm{S}$.J. Chung), R Dodel, I. Leroi, O. Monchi, and the other, anonymous, reviewer(s) for their contribution to the peer review of this work.

Publisher's note

Springer Nature remains neutral with regard to jurisdictional claims in published maps and institutional affiliations.

\section{RELATED LINKS}

MoCA: https://www.mocatest.org

(c) Springer Nature Limited 2021, corrected publication 202 